\title{
Assessing Probabilistic Wind Load Effects via a Multivariate Extreme Wind Speed Model: A Unified Framework to Consider Directionality and Uncertainty
}

\author{
Xinxin Zhang ${ }^{1}$, Xinzhong Chen $^{2}$ \\ ${ }^{1} \mathrm{PhD}$ Candidate, ${ }^{2}$ Professor, National Wind Institute, Department of Civil and Environmental \\ Engineering, Texas Tech University, Lubbock, TX, USA. \\ Emails: zhang.xinxin@ttu.edu; xinzhong.chen@ttu.edu
}

\begin{abstract}
This study presents a new approach of estimating wind load effects (responses) for various mean recurrence intervals (MRIs) with consideration of both directionality and uncertainty of wind speed and 11 wind load effects. The joint probability distribution model of directional extreme wind speeds is 12 established based on extreme wind speed data using multivariate extreme value theory with Gaussian 13 Copula. The distribution of yearly maximum wind load effect is then calculated through the exceeding 14 probability of directional wind speeds over the corresponding levels. The uncertainty of extreme response 15 conditional on wind speed and direction is further considered using the theorem of conditional probability. 16 The proposed analytical framework can be considered as an analytical formulation of the existing 17 approach based on historical directional wind speed data, but with an additional capability of accounting 18 for the uncertainty of extreme response conditional on wind speed and direction. It can also be regarded 19 as an extension of the existing fully probabilistic methods with an additional capability of accounting for 20 directionality. Applications of the proposed approach are presented and the results are compared with 21 those from the existing approach to demonstrate its accuracy. The characteristics of directionality factor 22 for wind load effects are discussed. Finally, the influence of uncertainty of extreme response conditional 23 on wind speed and direction is further examined.
\end{abstract}

24 Key words: Multivariate extreme wind speed distribution; Gaussian Copula; Extreme wind load effect; 25 Directionality; Mean recurrence intervals; Reliability. 


\section{Introduction}

The importance of considering wind directionality effect in estimating probabilistic wind load effects of structures has been well recognized. As the most unfavorable direction which produces the largest wind load and structural response under given wind speed does not necessarily align with the direction of the strongest wind, considerations of directionality effect of wind, aerodynamics and structural characteristics result in a reduction of response as compared to the analysis regardless of direction, i.e., the consideration of the worst case scenario. A directionality factor is often introduced to model this reduction effect (Simiu and Heckert 1998; Rigato et al. 2001; Laboy-Rodriguez et al. 2014). In ASCE 7 10 (ASCE 2010), a directionality factor of 0.85 is specified for buildings. This factor is only applicable to the load specified in code for which calibrations have been made.

Project-specific wind engineering studies usually take advantage of wind tunnel studies for a more accurate definition of wind loads, which is then combined with the use of state-of-the-art approaches for consideration of directionality effect. Several approaches have been developed in literature, some of which are based on parent distribution and the others on yearly maximum value distribution of mean wind speed. Previous studies have shown that the discrepancies of predictions from these approaches are often significant (e.g., ASCE 1999; Sadek 2005). The lack of specificity in the use of approaches for combining wind tunnel data with directional wind speed statistics has been discussed in a recent critique of ASCE 7 procedure (Simiu et al. 2013).

The random process crossing rate approach initially introduced by Davenport (1977) remains one of the popular methods in North America. Wen (1983 and 1984) carried out an extensive study of directionality effect using this crossing rate approach. An alternative formulation for the crossing rate analysis was presented by Lepage and Irwin (1985) with a consideration of the derivative of direction variable of wind speed. The accuracy of predictions from this approach can be affected by the inadequate description of the extreme wind statistics with the commonly used Weibull distribution as the parent distribution of directional wind speed (Simiu and Scanlan 1996; Irwin et al. 2005; Isyumov et al. 2014). A 
51 bi-modal Weibull distribution was proposed for an improved modeling of the parent distribution of mean 52 wind speed (Xu et al. 2008; Isyumov 2014).

53 Another approach estimates distribution of extreme wind effect using historical directional yearly 54 maximum wind speed data (Simiu and Filliben 1981; Simiu and Heckert 1998). Because this method is 55 implemented based on observation data series rather than an analytical formulation, it is not very 56 convenient for a parametric study to gain fundamental understanding on how directional characteristics 57 affect the reduction of response. The storm passage method introduced in Isyumov et al. (2003) follows a 58 similar scheme but directly uses the time series of mean wind speed and direction during storm passages 59 instead of directional yearly maximum wind speed data. This method is also not convenient to carry out 60 parameter studies as it is not formulated in an analytical form.

61 The sector-by-sector method is also often used due to its simplicity (Simiu and Filliben 2005; Irwin 62 et al. 2005). It determines the extreme response for a target mean recurrence interval (MRI) directly from 63 the extreme wind speeds at different directions. Two variants of this approach are adopted in practice: 1) 64 The wind speeds at various directions (sectors) with a target MRI of R years are firstly determined. The 65 corresponding extreme responses are quantified and their maximum over all sectors is considered as the 66 response for MRI of $\mathrm{R}$ years; 2) The cumulative distribution functions (CDFs) of annual maximum 67 responses at different sectors are determined from the yearly extreme value distributions of directional 68 wind speeds. The CDF of annual maximum response over all sectors is then estimated as a product of 69 these CDFs of directional extremes under the assumption that extremes of directional wind speeds are 70 mutually independent. Both predictions can be less accurate than the predictions that take the statistical 71 dependence of extremes of directional wind speeds into consideration. It is noted that all these approaches 72 for directionality effect treat the extreme response conditional on wind speed and direction as a 73 deterministic quantity rather than a random variable. Therefore, these approaches are unable to account 74 for the uncertainty and directionality in a unified framework.

75 It is reported that during a storm passage, the wind direction may vary about 120 degrees (e.g., Cook 76 1982). Therefore, the extreme wind speeds in neighboring sectors often have certain level of correlation. 
77 A slightly different method of using directional extreme winds in mixed wind climates (typhoon and non-

78 typhoon winds) was introduced in Matsui and Tamura (2005), where the storm correlation between 79 directions was also not taken full account. Itoi and Kanda (2002) extended the Gumbel's bivariate 80 distribution, equivalent to Gumbel copula, to four directional extreme wind speeds with dependence 81 structures given empirically by assuming an identical slope for all marginal Type I distributions. 82 Estimations of direction-free wind speed were made and compared based on such models for directional 83 maximum wind speeds.

84 The first fully probabilistic method that accounts for uncertainties of wind speed and wind load 85 effect conditional on wind speed was given by Cook and Mayne $(1979,1980)$ for extreme wind load 86 effects of rigid structures. This method is referred to as the first-order method as it neglects the possibility 87 of larger wind load effects produced by second and higher-order strongest winds in a year (Gumley and 88 Wood 1982; Harris 1982). A full-order method was proposed by Harris (1982 and 2005). Chen and 89 Huang (2010) introduced a refined full-order method, which is capable of dealing with any type of 90 asymptotic extreme value distribution, and can be used for both rigid and flexible structures. It should be 91 emphasized that these fully probabilistic methods are incapable of further accounting for the effect of 92 directionality. The probabilistic wind load effects have also been addressed in literature from different 93 perspectives where Monte Carlo simulations are often required for the estimations (Kareem 1987 and 94 1988; Bashor and Kareem 2009; Diniz et al. 2004; Diniz and Simiu 2005; Hanzlik et al. 2005).

95 With the motivation to diminish the difference in predictions among existing methods and to permit 96 parametric study with analytical formulations, a new unified framework to include both directionality and 97 uncertainty is proposed in this study for estimating wind load effects for various MRIs. In this study, the 98 directional extreme wind speed data are used to model joint probability distribution of directional extreme 99 wind speeds based on multivariate extreme value theory with application of Gaussian copula (Mikosch 100 2006). Copula has been a popular approach in multivariate extreme value analysis (Renard and Lang 2007; 101 Cherubini, et al. 2004), while its applications to wind engineering has not been fully explored (Ioi and 102 Kanda 2002; Scholzel and Friederichs 2008). The Gaussian copula, as one of the copula models, can be 


\section{8}

regarded as identical to the classic multivariate Gaussian translation model in formulation (Grigoriu 2009). However, the introduction of copula with the concept of standardizing correlated variables into uniform distribution margins enables the potential use of a variety of existing models for the wind directionality studies. The multivariate extreme wind speed models determined from wind speed and wind velocity pressures are compared. With the multivariate extreme model of directional wind speeds, the probability of exceeding a certain wind effect level is then calculated through the probabilities of exceeding directional wind speeds over corresponding levels. The uncertainty of extreme response conditional on wind speed and direction is further considered using the theorem of conditional probability. The proposed analytical framework can be considered as an analytical formulation of the existing approach based on historical directional wind speed data (Simiu and Filliben 1981; Simiu and Heckert 1998), but with an additional capability of accounting for the uncertainty of extreme response conditional on wind speed and direction. It can also be regarded as an extension of the fully probability methods (Cook and Mayne 1979; Harris 2005; Chen and Huang 2010) with an additional capability of accounting for directionality. Applications of the proposed approach are presented and the results are compared with those from the existing approach that is based on historical extreme wind data in order to demonstrate its accuracy. The characteristics of directionality factor for wind load effects as function of MRI are discussed. Finally, the influence of uncertainty of extreme wind response conditional on wind speed and direction is further examined.

\section{Methodology of the proposed approach}

\subsection{Modeling of extreme wind speed at each sector}

To model the joint distribution of directional extreme wind speeds with multivariate extreme value theory, the first step is to determine the extreme value distribution of wind speed in each sector. Extensive literatures are available for modeling the distribution of univariate extreme wind speed. The methods used include a direct use of univariate extreme value theory based on extreme wind speed data (e.g., Cook and Mayne 1979 and 1980; Harris 1999), Peaks-Over-Thresholds (POT) method (e.g., Holmes and Moriarty 
1999; Simiu and Heckert 1996; An and Pandey 2005) and average conditional mean exceedance (ACER) method (Naess and Gaidai 2009) which used wind speed data over a selected high threshold, and the approach based on crossing rate of random wind speed process (e.g. ASCE 1999), and others.

It is most straightforward to use yearly maximum wind speed data to derive yearly extreme value distribution when sufficient wind speed data are available. A general extreme value distribution (GEV) can be used with the CDF expressed as

$$
\Psi(x)=\exp \left\{-\left[1+\varepsilon\left(\frac{x-m}{\delta}\right)\right]^{-1 / \varepsilon}\right\}
$$

134 where $m$ and $\delta$ are location and scale parameters, respectively; and $\varepsilon$ is the shape parameter which 135 determines the upper bound. When $\varepsilon<0$ it has a limited upper bound at $m-\delta / \varepsilon$ and is called the 136 reversed Weibull distribution (Type III). When $\varepsilon>0$ and $\varepsilon=0$, it becomes Frechet (Type II) and 137 Gumbel (Type I) distribution, respectively, with infinite upper tails. Both Type I and III distributions have 138 been used in wind speed distribution (Cook 1982; Simiu and Scanlan 1996; Holmes and Moriarty 1999; 139 Chen and Huang 2010).

140 The CDF of Type I distribution can be derived from Eq.(1) by taking a limit $\varepsilon \rightarrow 0$ :

$$
\Psi(x)=\exp \left[-\exp \left(-\frac{x-m}{\delta}\right)\right]
$$

where the mean and standard deviation (STD) of Type I distribution are $m+\gamma \delta$ and $\delta \pi / \sqrt{6}$, respectively, where $\gamma=0.5772$ is Euler constant.

The GEV can be estimated through traditional statistical methods such as the maximum likelihood 144 method, least square method, method of moment, probability weighted moment (e.g., Castillo 2005). In 145 wind engineering, the method proposed by Gumbel (Gumbel 1958) is often used for Type I distribution. 146 The drawback of using yearly maximum wind speed, sometimes termed as global maxima, is the small 147 sample size which leads to large statistical error. Therefore, the use of block maxima, e.g., monthly 148 maximum or storm maximum wind speeds, can reduce the statistical error due to the increased sample size. When the block maximum is considered as independent and identically distributed with the CDF 
denoted as $\Psi_{b V}(v)$, the CDF of yearly maximum $\Psi_{V}(v)$ is then calculated as $\Psi_{V}(v)=\left[\Psi_{b V}(v)\right]^{n_{a}}$, where $n_{a}$ is the average number of blocks in a year, e.g., when monthly maximum is used, $n_{a}=12$.

152 Based on this assumption, the parameters of global maximum GEV distribution can be directly calculated 153 from those of block maximum as

$$
\varepsilon_{G}=\varepsilon_{B}, \quad \delta_{G}=n_{a}^{\varepsilon_{B}} \delta_{B}, \quad m_{G}=m_{B}+\delta_{B} \frac{n_{a}^{\varepsilon_{B}}-1}{\varepsilon_{B}}
$$

154 where the subscript $\mathrm{G}$ and $\mathrm{B}$ denote global and block, respectively. For Type I distribution, note that when $155 \varepsilon_{B} \rightarrow 0, n_{a}^{\varepsilon_{B}}-1 \rightarrow \varepsilon_{B} \ln \left(n_{a}\right)$, Eq.(3) becomes

$$
\varepsilon_{G}=\varepsilon_{B}=0, \quad \delta_{G}=\delta_{B}, \quad m_{G}=m_{B}+\delta_{B} \ln \left(n_{a}\right)
$$

156 It is also noted that yearly maximum wind velocity pressure, i.e., $Q=0.5 \rho V^{2}$, where $\rho$ is air 157 density, or the square of wind speed, i.e., $V^{2}$, referred to as $Q$-model, has also been used to model the 158 extreme wind distribution (Cook 1982; Harris 1982 and 2005; Chen and Huang 2010). It is based on the 159 premise that the extreme of wind velocity pressure or the square of wind speed approaches asymptotic 160 extreme value distribution faster than that of wind speed. However, a study of extreme wind speed 161 estimates in various U.S. cities did not support the use of $Q$-model based on Type I distribution as 162 compared to the predictions based on wind speed data, referred to as $U$-model (Simiu et al. 2001). It is 163 noted that when the yearly maximum wind velocity pressure or square of wind speed is assumed to follow 164 a Type I distribution, the wind speed turns to follow a Type III distribution. In this paper, both $U$ - and $Q$ 165 models are addressed.

\section{$166 \quad 2.2 \quad$ Modeling of joint probability distribution of directional extreme wind speeds}

167 The joint probability distribution of directional extreme wind speeds can be determined using multivariate extreme value theory. Multivariate extreme value theory concerns the characterization, 169 estimation and extrapolation of the joint tails of multidimensional extreme value distributions. It has been 170 utilized in a variety of applications from environmental impact assessment to financial risk management 171 (Beirlant et al. 2004; Castillo et al. 2005; de Haan and Ferreira 2006), but has not yet been extensively 
172 utilized for problems related to probabilistic wind load effects. Once the marginal distribution of each individual variable in terms of extreme wind speed in each direction is determined, it is then, for the purpose of modeling their dependence structure, transformed into a standard distribution which can be unit Frechet, exponential, Gumbel, reversed exponential margin (extreme value Weibull), or others (Coles and Tawn 1991). There is also one particular standard margin of interest, the uniform margin which corresponds to the concept of copula, a popular way to describe dependence structures of multivariate distributions(e.g., Mikosch 2006; Nelsen 2006). Loosely speaking, copula is a function that links a joint distribution to its marginal distributions as its name suggests, a copula "couples" a joint distribution to its univariate margins.

Consider the joint CDF of multivariate random variables $V_{1}, V_{2}, \ldots, V_{n}$ as $H\left(v_{1}, v_{2}, \ldots, v_{n}\right)$. Their marginal CDFs are $\Psi_{V_{1}}\left(v_{1}\right), \Psi_{V_{1}}\left(v_{2}\right), \ldots, \Psi_{V_{n}}\left(v_{n}\right)$. The multivariate random variables can be transformed into random variables $U_{1}, U_{2}, \ldots, U_{n}$ with uniform distributions on $[0,1]$ through the mapping of CDF functions, i.e., $u_{i}=\Psi_{V_{i}}\left(v_{i}\right),(i=1,2, \ldots n)$. It can be proved that there is always a unique function, $C_{\text {copula }}($.$) , called copula that relates the standard margins, if they are continuous, to the joint distribution$ as (Nelsen 2006)

$$
H\left(v_{1}, v_{2}, \ldots, v_{n}\right)=C_{\text {copula }}\left(\Psi_{V_{1}}\left(v_{1}\right), \Psi_{V_{2}}\left(v_{2}\right), \ldots, \Psi_{V_{n}}\left(\mathrm{v}_{\mathrm{n}}\right)\right)=C_{\text {copula }}\left(u_{1}, u_{2}, \ldots, u_{n}\right)
$$

Therefore, once marginal distributions are given, the joint distribution solely relies on the dependence structure defined by the copula. One of the widely used copula families is Archimedean copula which includes Clayton, Frank and Gumbel copulas (Nelsen 2006). These types of copulas are usually used in bivariate case because their copula functions are built through a generator function with a single parameter which implies the dependence. However, in the case of more than two variables, the single parameter is insufficient to represent the complication of dependence structure.

Another copular family is called elliptical copula which uses an existing multivariate joint distribution with a symmetric positive definite covariance matrix, such as multivariate Gaussian distribution and multivariate student $t$ distribution, to model the dependence structure. The name of 
elliptical family indicates the limitation of this copula that the dependence between any two standard margins can only be described by elliptical shaped contours. The use of covariance matrix provides sufficient parameters, i.e., correlation coefficients between any two margins, to model the dependence structure. The Gaussian copula is a member of the elliptical copula family and is defined as follows:

$$
C_{\text {copula }}\left(u_{1}, u_{2}, \ldots, u_{n}\right)=G_{n}\left(y_{1}, y_{2}, \ldots, y_{n}\right)
$$

200 where $y_{i}=\Phi^{-1}\left(u_{i}\right)=\Phi^{-1}\left(\Psi_{V_{i}}\left(v_{i}\right)\right)=g_{i}\left(v_{i}\right)$ is the underlying standard Gaussian variable with zero 201 mean and unit variance; $g_{i}$ is the translation function for $V_{i} ; \Phi$ is the CDF of standard Gaussian 202 distribution; and $G_{n}$ is the CDF of $n$-dimensional normal distribution with mean 0 and covariance matrix $203 \Sigma$ with $\Sigma_{i i}=1$ and $\Sigma_{i j}=\Sigma_{j i}=\rho_{i j}$, the correlation coefficient of $Y_{i}$ and $Y_{j}$, i.e., $\rho_{i j}=E\left[Y_{i} Y_{j}\right]$.

204 The correlation coefficient between $V_{i}$ and $V_{j}$, i.e., $\zeta_{i j}=\left(E\left[V_{i} V_{j}\right]-\mu_{v_{i}} \mu_{v_{j}}\right) / \sigma_{v_{i}} \sigma_{v_{j}}$, is related to 205 that of $Y_{i}$ and $Y_{j}$ as follows

$$
\sigma_{v_{i}} \sigma_{v_{j}} \zeta_{i j}+\mu_{v_{i}} \mu_{v_{j}}=\int_{-\infty}^{\infty} \int_{-\infty}^{\infty} g_{i}^{-1}\left(y_{i}\right) g_{j}^{-1}\left(y_{j}\right) \phi\left(y_{i}, y_{j} ; \rho_{i j}\right) d y_{i} d y_{j}
$$

206 where $\phi\left(y_{i}, y_{j} ; \rho_{i j}\right)$ is the joint probability density function (JPDF) of bivariate Gaussian distribution 207 with correlation coefficient $\rho_{i j} ; \mu_{v_{i}}$ and $\sigma_{v_{i}}$ are mean and STD of $V_{i}$. It is noted that $\zeta_{i j}$ is an increasing function of $\rho_{i j}$ whose range is $[-1,1]$. However, the upper bound, $\zeta_{i j}^{u}=1$ only if $\Psi_{V_{i}}=\Psi_{V_{j}}$ and the lower bound $\zeta_{i j}^{l}=-1$ needs an additional condition that the probability density function (PDF) $f_{i}=f_{j}$ of $210 \Psi_{V_{i}}=\Psi_{V_{j}}$ is an even function, otherwise $\zeta_{i j}^{l}>-1$ (Grigoriu 2007).

211 In the case where $\Psi_{V_{i}}\left(v_{i}\right)$ is Type I extreme value distribution, the translation function is then 212 calculated as

$$
g_{i}^{-1}\left(y_{i}\right)=\mu_{v_{i}}+\sigma_{v_{i}} \frac{\sqrt{6}}{\pi}\left(-\ln \left\{-\ln \left[\Phi\left(y_{i}\right)\right]\right\}+\gamma\right)
$$

213 and Eq.(7) gives 


$$
\zeta_{i j}=\frac{6}{\pi^{2}} \int_{-\infty}^{\infty} \int_{-\infty}^{\infty}\left(-\ln \left\{-\ln \left[\Phi\left(y_{i}\right)\right]\right\}+\gamma\right)\left(-\ln \left\{-\ln \left[\Phi\left(y_{j}\right)\right]\right\}+\gamma\right) \phi\left(y_{i}, y_{j} ; \rho_{i j}\right) d y_{i} d y_{j}
$$

Obviously, the value of $\zeta_{i j}$ relies only on $\rho_{i j}$ and is irrelevant to the parameters of Type I distribution as shown in Fig.1. The difference between the correlation coefficient of two Type I variables and their underlying Gaussian variables is small, although the translation function has its limit in negatively correlated case, e.g., the lower bound $\zeta_{i j}=-0.89$ corresponds $\rho_{i j}=-1$.

The CFD of extreme wind speed regardless of wind direction can be then determined as

$$
\Psi_{V}(v)=\operatorname{Pr}\left(V_{1} \leq v, V_{2} \leq v, \ldots, V_{n} \leq v\right)=H(v, v, \ldots, v)=G_{n}\left(g_{1}(v), g_{2}(v), \ldots, g_{n}(v)\right)
$$

From the properties of copula, the upper and lower bounds of $\Psi_{V}(v)$ can be determined as (Nelsen 2006; Grigoriu 2007):

$$
\max \left\{\left[1-n+\sum_{i=1}^{n} \Psi_{V_{i}}(v)\right], 0\right\} \leq \Psi_{V}(v) \leq \min \left\{\Psi_{V_{1}}(v), \Psi_{V_{2}}(v), \ldots, \Psi_{V_{n}}(v)\right\}
$$

where upper bound corresponds to the case of fully correlated margins.

It is noted that the product of each marginal $\operatorname{CDF} \Psi_{V, i n d}(v)=\prod_{i=1}^{n} \Psi_{V_{i}}(v)$, i.e., the case of independent margins, is also a special copula function which must be included within such bounds in Eq. ( 11 ) (Grigoriu 2007). If $\Psi_{V}(v) \geq \Psi_{V \text {,ind }}(v)$, it is called positively quadrant dependent. Otherwise, it is negatively quadrant dependent. Moreover, an important property of a multivariate Gaussian distribution is that for $G_{n}$ and $G_{n}^{\prime}$ with covariance matrix $\Sigma$ and $\Sigma^{\prime}$, where $\Sigma_{i j} \geq \Sigma_{i j}^{\prime}$ for any $i \neq j$, then $G_{n}\left(y_{1}, y_{2}, \ldots, y_{n}\right) \geq G_{n}^{\prime}\left(y_{1}, y_{2}, \ldots, y_{n}\right)$, (e.g., Grigoriu 2007). This property implies that the estimation of wind load effect based on the independence assumption of directional wind speed is always conservative if correlation coefficients between directions are non-negative. Consider the case of bivariate Gaussian distribution $G_{2}\left(y_{1}, y_{2}\right)$, the fractile value of $y_{R}$, where $G_{2}\left(y_{R}, y_{R}\right)=1-1 / R$, is affected by the correlation coefficient $\rho_{i j}$. Fig.2 shows the change of $y_{R}$ as a function of correlation coefficient, where $y_{R 0}$ is the estimate with $\rho=0$, and $R=50$ and 500. It is evident that the assumption of independence 
results in conservative estimation of $y_{R}$ in the case of positive correlation, while the negative correlation

has almost no influence on $y_{R}$. That is, when the correlation coefficients are not all non-negative, the negative correlation coefficients can be considered as zero for a good approximation. Therefore, with the use of Gaussian copula, the calculation based on the independent assumption can roughly be considered to be conservative although the calculation based on the left hand side of Eq. (11) is strictly conservative.

\subsection{Distribution of extreme wind load effect}

For a given structure under a prescribed wind condition, the dynamic wind loads are quantified through wind tunnel or full-scale measurements or analytical loading models. The extreme response within an hour for a given wind speed and direction is determined through dynamic response analysis or measurements. The wind load effect is affected by wind characteristics, aerodynamic and structural properties. The extreme wind load effect in the $i$-th wind direction $(i=1,2, \ldots, n)$ is expressed as

$$
X_{i}=\frac{1}{2} \rho V_{i}^{2} C_{i}\left(V_{i}\right)
$$

where $V_{i}$ is hourly mean wind speed at $i$-th direction; and $C_{i}\left(V_{i}\right)$ is normalized extreme load effect (response) coefficient within one hour or simply termed as wind load coefficient. In the case of rigid structures, the wind load coefficient $C_{i}\left(V_{i}\right)$ can be independent of mean wind speed. On the other hand, it is a function of mean wind speed for flexible structures due to dynamic amplification effect. In general, $C_{i}\left(V_{i}\right)$ is a random variable. Its uncertainty can be attributed to aleatory uncertainty and epistemic uncertainty which are inherited from the randomness of the influence of terrain, aerodynamic and structural dynamic effects. At strong wind conditions, the air density can be regarded as deterministic (e.g., Harris 1982 and 2005).

When the load coefficients $C_{i}\left(V_{i}\right)(i=1,2, \ldots, n)$ are considered as deterministic values as did in all existing approaches for consideration of directionality, the CDF of annual maximum extreme wind load effect considering all directions can be readily estimated from the joint CDF of directional extreme wind speeds: 


$$
\Psi_{X}(x)=H\left(v_{x 1}, \ldots, v_{x n}\right)
$$

where $v_{x i}(i=1,2, \ldots n)$ is the wind speed at $i$ th direction corresponding to extreme response level $x$ determined by Eq.( 12 ), i.e., $v_{x i}=\sqrt{2 x / \rho C_{i}\left(v_{x i}\right)}$.

When $C_{i}\left(V_{i}\right)(i=1,2, \ldots, n)$ are considered as random quantities, the $\operatorname{CDF} \Psi_{X}(x)$ is then estimated as follows using the theorem of conditional probability:

$$
\Psi_{X}(x)=\int \ldots \int \mathrm{H}\left(v_{x 1}\left|c_{1}, \ldots, v_{x n}\right| c_{n}\right) f_{C}\left(c_{1}, \ldots, c_{n}\right) d c_{1} \ldots d c_{n}
$$

where $f_{C}\left(c_{1}, \ldots, c_{n}\right)$ is the JPDF of $C_{i}\left(V_{i}\right)(i=1,2, \ldots, n)$. The extreme wind load effect coefficients $C_{i}\left(V_{i}\right)(i=1,2, \ldots, n)$ depend on the terrain conditions and on aerodynamic and structural dynamic effects for the structure being considered. They can be correlated in general, but are considered to be less correlated than the parent wind effect processes. If $C_{i}\left(V_{i}\right)(i=1,2, \ldots, n)$ are assumed to be mutually independent, then $f_{C}\left(c_{1}, \ldots, c_{n}\right)=f_{1}\left(c_{1}\right) f_{2}\left(c_{2}\right) \ldots f_{n}\left(c_{n}\right)$, where $f_{i}\left(c_{i}\right)$ is the PDF of $C_{i}\left(V_{i}\right)$. Technically, Eq.(14) can be evaluated through Monte Carlo simulation, i.e., by first generating samples of $c_{i}(i=1,2, \ldots, n)$, and then calculating $\mathrm{H}\left(v_{x_{1}}\left|c_{1}, \ldots, v_{x n}\right| c_{n}\right)$, followed by an ensemble average of these estimations. From $\Psi_{X}(x)$, the wind load effects for various MRIs are determined.

An alternative formulation to Eq. (14) is also provided as:

$$
\Psi_{X}(x)=\int \ldots \int F_{C}\left(c_{1}\left|v_{x 1}, \ldots, c_{n}\right| v_{x n}\right) h\left(v_{x 1}, \ldots, v_{x n}\right) d c_{1} \ldots d c_{n}
$$

270 where $F_{C}\left(c_{1}\left|v_{x 1}, \ldots, c_{n}\right| v_{x n}\right)$ is the JCDF of $C_{i}\left(V_{i}\right)(i=1,2, \ldots, n)$ and $h\left(v_{x 1}, \ldots, v_{x n}\right)$ is the JPDF of 271 directional extreme wind speed with given $v_{x 1}, \ldots, v_{x n}$. Eq.(15) can also be evaluated through Monte 272 Carlo simulation. Compared to Eq.(14), when $C_{i}\left(V_{i}\right)(i=1,2, \ldots, n)$ are assumed to be mutually 273 independent, Eq.(15) is computationally more efficient because the computation effort of $274 F_{C}\left(c_{1}\left|v_{x 1}, \ldots, c_{n}\right| v_{x n}\right)$ is much less than that of $\mathrm{H}\left(v_{x 1}\left|c_{1}, \ldots, v_{x n}\right| c_{n}\right)$.

The proposed new approach has the following advantages: a) it can consider the effects of both uncertainty and directionality; and b) it facilitates a systematic parametric study as the method is 
presented in analytical formulation. The proposed approach is formulated based on the analytical

directional wind speed model, which establishes the relationship between exceeding probabilities of wind load effect and its corresponding directional wind speeds. Essentially, this approach not only combines the statistical wind climate information and wind tunnel testing data, but also allows the two parts of work to be carried out separately by different groups of experts. In another word, when an accurate directional wind speed model is established, the distribution of extreme wind load effect can be derived without using the original wind speed data. Furthermore, when multiple wind load effects under the same directional wind climate condition are of concern, the advantage of the proposed approach is even more significant than using the original wind speed data for the analysis of each wind effect.

\section{Validation and Discussion}

In this section, this new approach will be validated for the case where the directional extreme wind load coefficients are treated as deterministic values. The proposed method is expected to result in consistent predictions as the existing method based on historical observation data of directional extreme wind speed (Simiu and Filliben 1981; Simiu and Scanlan 1996). Discussions on extreme wind load effects for various MRIs and directionality factor are made with the use of $U$ - and $Q$-models. It is followed by discussions where the directional wind load coefficients are further treated as random quantities.

\subsection{Directional extreme wind speed model}

\subsubsection{Wind speed observation data}

The directional hourly mean wind speed data used in this study was processed based on the data set 6405 from ASOS (Automated Surface Observation System, NOAA) at Baltimore, MD, USA, dated from January $1^{\text {st }}, 2000$ to August $31^{\text {st }}, 2012$. The raw data contains one-minute mean wind speed reported in unit of knot and a wind direction with a resolution of 1 degree. The measurement height is at $33 \mathrm{ft}(10$ meters) throughout the time of observation. The hourly mean wind speed and direction is then calculated 
using a vector average algorithm. After post-processing, the mean wind speed is in unit of miles per hour

(mph) and wind direction has a resolution of 1 degree. Then, for simplicity, these directional hourly mean wind speeds are partitioned into eight directional sectors, each of which swept an area from $\alpha_{i}-22.5$ degrees to $\alpha_{i}+22.5$ degrees and are denoted by their central directions $\alpha_{i}=45, \ldots, 315,360$ degrees, representing directions of $\mathrm{NE}, \mathrm{E}, \ldots, \mathrm{N}$. When a particular problem is sensitive to direction change, a finer resolution of directional sector can be used, while the uncertainty in the modeling of each directional wind speed increases due to reduced number of samples. It should also be noted that there is a directional wind speed "masking" issue where the wind speeds at a fixed hour in all directions other than the reported direction are recorded as zero. A simple procedure was used for the treatment of such issue (Coles and Walshaw 1994). The influence of the treatment to the "masking" problem has been addressed in another literature (Zhang 2015), while in this paper it is not discussed in detail because the focus of this paper is to introduce the proposed methodology for given wind speed data and wind tunnel test results.

The monthly maximum hourly mean wind speed data are then obtained for each month at each directional sector. In each direction, there are altogether 152 monthly maxima available. However, two of them were possibly affected by hurricanes according to historical climate information and thus were removed because they may have different statistical properties as compared to those produced by nonhurricane phenomenon. Therefore, we have 150 monthly maximum mean hourly wind speed data within each direction and they can be loosely assumed to be identically independently distributed. The use of monthly maxima as samples of each directional variable enables the pair-wise calculation of covariance matrix of directional wind speeds. The non-directional wind speed data is selected from the maximum wind speed from all directions within each month.

\subsubsection{Extreme wind speed model from $U$-model}

As shown in Fig. 3, both Type I and GEV distributions are used to fit directly to the monthly maximum wind speed data in each direction, i.e., $\Psi_{V_{i}}$ represented by black dashed lines, and to the nondirectional data, i.e., $\Psi_{V_{n o n}}$ represented by red solid lines with circle markers. Also, the CDF of 
directionless wind speed, i.e., $\Psi_{V}$ represented by black solid lines with triangle markers, is calculated using the proposed multivariate model with the marginal distribution $\Psi_{V_{i}}$ obtained above, i.e., using Eqs.

(6) to (10). The 50- and 500-year wind speeds at different directions are displayed in Table 1 to show the directionality characteristics of wind speed.

First, the difference between the uses of Type I and GEV margins is discussed. From Table 1, for wind speed in each direction, the use of Type I margins yields larger predictions in most directions as compared to the use of GEV margins in this example because most GEVs have negative shape parameters. The shape parameter not only influences the absolute values of predictions but also their directionality characteristics. For example, for MRI = 50 years, the NW direction is the second largest direction for Type I margins, whilst it is the fourth largest for GEV margins. Moreover, with the use of GEV margins, the directionality characteristics of wind speed tends to change with the change of MRI, e.g., when MRI increases from 50 years to 500 years, the corresponding wind speed in SE direction grows from the fifth largest to the second largest and will eventually be the most dominant direction for even larger MRI.

The directionless wind speed can be predicted from both $\Psi_{V_{n o n}}$ and $\Psi_{V}(v)$. When MRIs are low, e.g., no more than 500 years, with the use of a given margin, both distributions are close to each other in prediction as can be seen from Fig. 3. Also, from either distribution, the use of Type I distribution for non-directional wind speed data also yields larger prediction compared to GEV distribution, e.g., from $\Psi_{V_{n o n}}$, the 50- and 500-year wind speeds regardless of direction are 65.5 and $78.1 \mathrm{mph}$ estimated from Type I distribution, and 55.5 and $59.3 \mathrm{mph}$ from GEV, respectively. It is also noted that when GEV margins are used for calculating $\Psi_{V}$, the extrapolation at large MRIs, e.g., larger than 500 years, may be unrealistic attributed to the its sensitivity to the shape parameter and due to the fact that $\Psi_{V}(v)$ follows the trend of one of the marginal distribution which has a lower probability of exceedance, i.e., the direction of dominant wind speed. As shown in Fig. 3(b), when MRI $>500$ years, $\Psi_{V}$ start to follow the 349 trend of a dominant wind direction which has Type II distribution of wind speed. 
The multivariate wind speed model is a consistent modeling between margins and directionless wind

speed. The property that $\Psi_{V}(v)<\Psi_{V_{i}}(v)$ (for any $\left.i=1,2, \ldots, 8\right)$ ensures that the directionless wind speed estimated from the multivariate model is always larger than any of the directional wind speeds for any MRI level (Nelson 2006). It is also proved that, theoretically, $\Psi_{V}(v) \neq \Psi_{V_{\text {non }}}(v)$ unless the marginal distributions are identical (Grigoriu 2007).

Denote the R-year wind speed determined from $\Psi_{V_{\text {non }}}$ and $\Psi_{V_{i}}$ as $v_{R, \text { non }}$ and $v_{i, R}$ respectively. Theoretically $\max \left(v_{i, R}\right)<v_{R, \text { non }}$. However, it is observed that there is a possibility that $\max \left(v_{i, R}\right)>$ $v_{R, \text { non }}$, i.e., the predicted wind speed from $\Psi_{V_{\text {non }}}$ at a certain MRI can be exceeded by one or more directional wind speeds at the same MRI level. Such an inconsistency is attributed to the modeling uncertainty associated with limited numbers of wind speed samples. The calculated $v_{R, n o n}$ and $v_{i, R}$ are the estimates of their true values with uncertainty. The uncertainty, which is often represented by an confidence interval around its true value, increases for a larger MRI. It is possible that a sample estimate gives $\max \left(v_{i, R}\right)>v_{R, n o n}$. Such inconsistency will result in some difficulties in later applications such as determination of the directionality factor. Therefore, the discussions followed will be based on the multivariate wind climate model with Type I margins, where the distribution of wind speed regardless of direction is calculated from the multivariate wind climate model without the above inconsistency.

Fig. 4 shows the typical dependence structure patterns between $\mathrm{W}$ direction and other two directions, i.e., E and NW, in terms of monthly wind speeds whose margins are assumed to be Type I distribution and their underlying Gaussian variables. The use of GEV margins would result in almost identical patterns in this example and thus was not shown for the sake of brevity. Clearly, the dependence patterns 370 of the underlying variables are all elliptical which implies that the Gaussian copula is an appropriate 371 model for directional extreme wind speeds. 
Table 2 shows the covariance matrix calculated directly from monthly maximum wind speed and underlying Gaussian variables. The covariance matrix of the underlying Gaussian variables can also be calculated numerically from the non-Gaussian covariance matrix by using Eq.( 7 ) or ( 9 ). The largest correlation coefficient is around 0.8 , and the wind speeds are mostly correlated to their adjacent directions. It can be proved that the covariance matrix of monthly maximum variables is equal to that of the yearly maximum variables given that both follow the same type of distribution, i.e., either Type I or GEV distribution. Accordingly, the corresponding Gaussian variables from monthly extreme and yearly extreme wind speeds also have the same covariance matrix.

\subsubsection{Extreme wind speed model from $Q$-model}

To determine extreme wind speed model from $Q$-model, a change of variable from $V$ to $Q=V^{2}$ is carried out and same analysis is repeated. The distributions of $Q_{i}$ in each direction, $\Psi_{Q_{i}}\left(q_{i}\right)$, and the distribution of directionless $Q, \Psi_{Q}(q)$, are plotted in Figs. 5(a) and 5(b) for Type I and GEV margins, respectively. Similar to $U$-model, the use of GEV margins leads to a result largely influenced by the shape parameters and is more deviated from the prediction with non-directional data, $\Psi_{Q_{n o n}}(q)$, compared to the use of Type I margins. The predicted wind speed for MRI = 50 and 500 years are 58.9 and $66.5 \mathrm{mph}$ from $Q$-model which are $9.9 \%$ and $14.9 \%$ less than those from $U$-model. The 50 - and 500-year wind speeds in each direction derived from $Q$-model are also summarized in Table 1 . The predictions from $Q$-model are generally smaller than the ones from $U$-model using the same margins. Also the results from Type I $Q$ model are close to the ones from GEV $U$-model. The use of $Q$-model offers an alternative modeling technique in case the predictions of $U$-model are too conservative. The difference between wind speed models derived from $Q$-model and $U$-model will be further inherited in the estimation of wind load effect as will be discussed in the following section. 


\subsection{Variation of directional wind load effect coefficient}

The wind load effect coefficient at a given wind speed $v_{i}$ and direction $\alpha_{i}$ is given as a power function of wind speed:

$$
C_{i}=v_{i}^{b-2} C_{0 i} C_{\alpha i}
$$

398 where $C_{0 i}$ is generally a random quantity with a mean $E\left(C_{0 i}\right)=C_{0}$, which remains the same for all 399 directions, and with a coefficient of variation, $V_{c i}$, which reflects the uncertainty of extreme wind load 400 coefficient in the $i$-th direction and may take different value for each direction; $C_{\alpha i}$ is a deterministic 401 value for the $i$-th direction and can be different for each direction, which reflects the directionality 402 characteristics of wind load coefficient, and $\max \left\{C_{\alpha i}, \mathrm{i}=1,2, \ldots, \mathrm{n}\right\}=1 ; b$ is a power law exponent 403 indicating the growth rate of the wind load effect with wind speed, and $b=2$ in the case of rigid 404 structures and $b>2$ for flexible structures due to dynamic amplification effect. In this extreme wind 405 effect model, its mean value varies with wind speed but its coefficient of variation is assumed as not a 406 function of wind speed. It is noted that other extreme wind effect models can also be used, for example, replacing the power function of wind speed with other functions and a consideration of the coefficient of variation as a function of wind speed.

For comparison and validation purposes, $C_{0 i}$ is first treated as a deterministic value $\left(V_{c i}=V_{c}=0\right.$ for all directions) and we borrow the example from Table 8.1.1 in Simiu and Scanlan (1996) as $C_{\alpha \mathrm{i}}(i=$ $1,2, \ldots, 8)$ shown in Table 3 denoted as Example 1, where $C_{0 i}=C_{0}=3.33$. The corresponding 50- and

412500 -year wind load effects at each direction of both $b=2$ and 3 are calculated as $x_{i, R}=\frac{1}{2} \rho v_{i, R}^{b} C_{0} C_{\alpha i}$, 413 where $v_{i, R}$ is the R-year wind speed from the $i$ th direction. All the calculated wind load effects are 414 normalized by the 500-year estimate without consideration of directionality, i.e., the worst case estimate $415 x_{w, 500}=\frac{1}{2} \rho v_{500}^{b} C_{0}$ and the results are summarized in Table 3. Clearly, there are two dominant directions 416 for rigid structures with $b=2$, i.e., $\mathrm{E}$ and $\mathrm{W}$ directions. The second dominant direction, i.e., $\mathrm{W}$ direction, 417 becomes more significant for wind load effects of flexible structures with $b=3$. Another example of $C_{\alpha i}$, 
denoted as Example 2, is generated based on the directional character of one of the generic typical

\subsection{Comparison and validation of wind load effect estimations}

To verify the accuracy of the proposed approach, calculation from the existing approach based on historical extreme wind speed data (Simiu and Filliben, 1981; Simiu and Scanlan, 1996) is also carried out and compared with the results of the proposed approach. With the existing approach, the historical monthly maximum directional wind speed data are used instead of yearly maximum data. First, using the monthly maximum wind speed record, the corresponding wind load effect at each direction of the month is calculated. The largest value among all directions is chosen as the maximum wind load effect of the month. These monthly maximum wind load effect samples, $X_{m}$, which can be termed as directionless wind load effect samples, are then converted in terms of equivalent wind speed samples, $V_{\text {eqm }}=$ $\left(2 X_{m} / \rho C_{0}\right)^{1 / b}$, and fitted into Type I extreme value distribution denoted as $\Psi_{m V_{e q}}(v)$. The yearly maximum distribution of equivalent wind speed is obtained through $\Psi_{V_{e q}}(v)=\left[\Psi_{m V_{e q}}(v)\right]^{12}$, and the yearly maximum distribution of wind load effect is related to it as $\Psi_{X}(x)=\Psi_{V_{e q}}\left(v_{x}\right)$ where $v_{x}=$ $\sqrt{2 x / \rho C_{0}}$ and the wind load effects for various levels of MRIs are then determined. As the equivalent wind speed follows Type I distribution, the yearly maximum wind load effect follows a Type II distribution. The use of equivalent wind speed in fitting the extreme value distribution is consistent with the use of $U$-model wind climate in the proposed approach. On the other hand, if Type I distribution is fitted to the square of $V_{e q m}$, i.e., $Q_{e q m}=\left(V_{e q m}\right)^{2}$, results from the existing approach corresponds to the use of $Q$-model in the proposed approach.

Figs. 6(a) and 6(b) show the comparison of predicted yearly maximum distribution of wind load effect using both approaches for rigid and flexible structures, respectively. It is observed when $U$-model is used, the estimations of wind load effect from both approaches are close to Type II distributions which 441 makes the prediction for larger MRIs noticeably conservative as compared to the results from $Q$-model. 
As summarized in Table 4, for rigid structures as shown, i.e., $b=2$, the predictions of normalized wind

load effect from the proposed approach, i.e., $x_{R} / x_{w, 500}$, are 0.346 and 0.504 for $R=50$ and 500 years using $U$-model, and 0.266 and 0.340 using $Q$-model, which are $23.1 \%$ and $32.6 \%$ less. For flexible structures, due to a larger $b$ value, the corresponding differences are further enlarged.

The predictions from two approaches, i.e., existing approach and the proposed approach, are in good agreement in general as seen in Fig. 6 and Table 4. Relatively larger discrepancies are observed in the calculation using $U$-model. For rigid structures, the differences of predictions are $6.7 \%$ and $8.3 \%$ for MRI $=50$ and 500 years, respectively. For flexible structures, the difference is enlarged because $b>2$, e.g., $10.2 \%$ and $12.7 \%$ for MRI $=50$ and 500 years. Such differences are mainly inherited from difference in the directional wind climate model. In the existing approach, the directional wind speed model is reflected in the wind speed data, while for the proposed approach, it is described by the fitted analytical model. It is expected that the predictions from both approaches will become more agreeable when both approaches use an identical wind climate model, particularly with sufficient data.

To verify this point, 6,000 monthly maximum directional wind speed data are generated randomly based on the Gaussian copula model. These data are then used in the existing approach and the resulting distribution of yearly maximum wind load effect is compared with that of the proposed approach. The results are shown in Fig. 7. The predictions from both approaches for both rigid and flexible structures are almost identical although the minor difference resulted from the errors added up from fitting of the wind speed in each direction in the proposed method compared to the one-time fitting of $V_{e q m}$ or $Q_{e q m}$ in the existing method. This means that the discrepancy of both approaches raised from the statistical error can be diminished given sufficient data.

In real engineering practice, there is usually less data available than what is needed. To further investigate the discrepancy between these two approaches under lack of data, the following study is carried out. First, 150 directional monthly maximum wind speed are simulated from the analytical wind climate model and used for calculating a sample of $\Delta X=\left(X_{E}-X_{P}\right) / X_{E}$, where $X_{E}$ and $X_{P}$ are the 
predicted wind load effects from the existing and the proposed approaches, respectively. Then the

statistics of $\Delta X$ are obtained by repeating the experiment 1000 times. As shown in Fig.8 in terms of $95 \%$ confidence interval, i.e., $1.96 \mathrm{STD}$ around the mean, the average $\Delta X$ is very small and close to 0 , e.g., $2 \%$ and $3 \%$ for 50 - and 500-year wind load effects when $b=2$; and $2.5 \%$ and $3 \%$ when $b=3$. This is consistent with the difference shown in Fig. 7. The 95\% confidence interval indicates the two approaches are very likely to be within a maximum absolute difference of $8.9 \%$ and $11.3 \%$ for 50 - and 500 -year wind load effect when $b=2$. These increase to $14.3 \%$ and $18 \%$ when $b=3$. The confidence interval should be narrower given a larger sample size.

\subsection{Directionality factor}

The directionality factor $K_{d}$ is defined as the ratio of the predicted wind load effect with consideration of directionality to that of the worst case as $K_{d}=x_{R} / x_{w, R}$. The value of $K_{d}$ is always less than one since $v_{\mathrm{R}}>v_{i, \mathrm{R}}$ for any direction according to the multivariate wind speed model. In ASCE7-10 standard, $K_{d}=0.85$ is used as independent of MRI for simplicity. In Rigato et al. (2001), $K_{d}$ increases when MRI increases from 50 to 500 years, while in Simiu and Filliben (1981), $K_{d}$ may decrease or increase as MRI increases from 20 to 50 years.

For Example 1 considered in this study, Fig. 9 shows the directionality factor as a function of MRI estimated from $U$ and $Q$-models for $\mathrm{b}=2$ and 3. It is observed that $K_{d}$ remains almost unchanged for various MRIs. Although the absolute values of estimate from $Q$-model are much different from those from $U$-model, their corresponding $K_{d}$ are almost the same. For $\mathrm{b}=2$, the wind load effect at $\mathrm{E}$ direction is more dominant, while for $\mathrm{b}=3$, both wind load effects at $\mathrm{E}$ and $\mathrm{W}$ directions become dominant. Accordingly, $K_{d}$ is lower in the case of $\mathrm{b}=3$.

To further understand the characteristics of directionality factor, another case study in terms of different directional wind load coefficient, namely Example 2 as shown in Table 3, but under the same wind speed model is considered. As shown Table 3, the directionality characteristics of wind load effect in terms of 50- and 500-year wind load effects at various directions show almost no change despite the 
changes in MRI, parameter $b$ and wind speed models used. In all cases within this example, the wind load effect at $\mathrm{W}$ direction is exceptionally dominant. As expected, the estimated $K_{d}$ remains almost constant accordingly. In general, the directionality factor is determined by the directionality characteristics of wind speed and wind load effect coefficients, thus can vary with MRI and parameter $b$ (e.g., Isyumov et al. 2014).

\subsection{Consideration of uncertainties of wind load coefficients}

The above calculations are all based on the assumption of deterministic directional wind load coefficients. Their uncertainties can be further accounted using the proposed approach. The following analysis is based on $U$-model. First, consider a simplified case where the uncertainties of wind load coefficients are independent of direction and wind speed. The mean wind load coefficients as functions of direction, $C_{\alpha i}(i=1,2, \ldots, 8)$, are the same as the two examples shown in Table 3, but $C_{0 i}$ in Eq. ( 16 ) is now regarded as a random variable, which is assumed to follow a log-normal distribution with a mean $E\left(C_{0 i}\right)=C_{0}$ and coefficient of variation $V_{c i}$.

The distribution of wind load effect considering both directionality and uncertainty is calculated by Eq.(14). For this simplified case with only one random variable, the calculation can be implemented using numerical integration while Monte Carlo simulation procedure is more efficient especially when multiple random variables are involved. When the Monte Carlo procedure is used, a random number $C_{0 i}$ is first generated for each direction, then the distribution of wind load effect is determined using the aforementioned framework. The procedure is repeated for 1000 times resulting 1000 distributions and their ensemble average is considered as the results with consideration of both directionality and uncertainties. In addition, the distributions of worst case wind load effect are also calculated each time and the corresponding ensemble average represents its counterpart with consideration of only uncertainties. This prediction is same as that from the fully probabilistic method.

Table 5 summarizes predicted 50- and 500-year wind load effects with consideration of uncertainty of load effect coefficients, which are normalized by 500 -year worst case wind load effect, $x_{w, 500}$, 
determined under $V_{c i}=0$. First, it is assumed that the coefficient of variation of wind load coefficient is the same for each direction, i.e., $V_{c i}=V_{c}$ where $V_{c}$ is a constant. It is observed that the increase of the variations of load effect coefficients brings increase to the predicted wind load effect. The distributions of worst case wind load effects estimated for a rigid structure of Example 1 are shown in Fig. 10(a) with various $V_{c}$ levels for illustration purpose. The increases of the 500-year wind load effect are 2.2, 10.3, 20.7 and $40.1 \%$ for $V_{c}=0.1,0.2,0.3$, and 0.4 , respectively, from that for $V_{c}=0$, i.e., without consideration of uncertainty. The predictions with consideration of both directionality and uncertainties are shown in Fig. 10(b). The increase of the 500-year wind load effect due to consideration of uncertainty are 2.2, 10.3, 21.2 and 41.3 for $V_{c}=0.1,0.2,0.3$, and 0.4 , respectively. Such increase is an accumulation of the increased wind load effects in each direction due to the corresponding variation of wind load coefficient. Although increasing the coefficient of variation of load effect coefficient results in increase of the absolute wind load effect, it may or may not lead to a change in $K_{d}$. As shown in Fig.11(a), $K_{d}$ does not change much for this case study. Calculations for Example 2 are also carried out. As shown in Fig.11(b), $K_{d}$ shows an obvious decrease in this example when $V_{c}$ increases.

When the variation of load effect coefficient is a function of direction, its influence on $K_{d}$ can be more obvious. Instead of using the constant $V_{c}$ for all directions in the previous examples, various $V_{c i}$ values were assigned to each direction. Two cases were calculated and compared as $V_{c 1}(\alpha)=\left[V_{c i}\right]=$

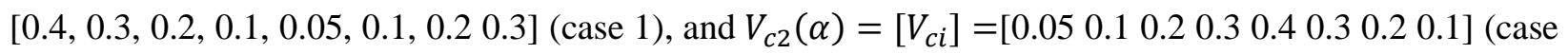
2), for directions E, NW, .., SE, respectively. The predicted distributions of yearly maximum wind load effect are plotted in Fig. 12. These predictions include those without consideration of both uncertainty and directionality, with consideration of only uncertainty, and with consideration of both uncertainty and directionality. The 50- and 500-year wind load effects are summarized in Table 5. The corresponding $K_{d}$ is shown in Fig.11. Obvious changes in $K_{d}$ can be seen in both cases because the percentage of increase of wind load effect in each direction differs largely from each other due to different $V_{c i}$ value assigned. 
The value of $K_{d}$ is in fact the ratio of predictions with and without consideration of directionalilty

effect, which is associated with how the directionality of wind speed and the directionality of wind load coefficient are combined. The uncertainty of wind load coefficient may or may not change the value of $K_{d}$ significantly depending on whether or not the consideration of uncertainty in directional wind load coefficients noticeably alters the directionality characteristics.

\section{Conclusion}

This study presented a new approach of considering both uncertainty and directionality for estimating wind load effects for various MRIs. This approach was based on the multivariate directional extreme wind speed distribution model which was established using multivariate extreme value theory with Gaussian copula. The proposed approach is an extension of existing approaches with a new capability to account for both uncertainty and directionality, which had been impossible within current existing approaches. The proposed approach also facilitates comprehensive parameter studies as the prediction is based on analytical formulation rather than a direct use of wind speed observation data.

Applications of the proposed approach presented in this study illustrated its accuracy through comparisons between its results and those from the existing approach based on historical wind speed data. The directional extreme wind speed models developed using wind speed data and using the square of wind speed, referred to as $U$ - and $Q$-models, respectively, were discussed. The example wind speed data were better fitted by $Q$-model whereas the $U$-model was more conservative. For both $U$ - and $Q$-models, Type I distribution was a more appropriate marginal distribution in building multivariate wind climate model than GEV distribution which was largely influenced by the shape parameter. With the proposed approach, the characteristics of directionality factor were investigated, which was affected by the directionality of wind speed and wind load effect. The influence of the uncertainty of wind load effect coefficients was further investigated which not only affected predicted extreme wind load effects but also 564 the directionality characteristics. 


\section{Acknowledgements}

The support for this work provided in part by NSF Grant No. CMMI-1029922 is greatly

\section{References}

An, Y., and Pandey, M. D. (2005). "A comparison of methods of extreme wind speed estimation." Journal of Wind Engineering and Industrial Aerodynamics, 93(7), 535-545.

ASCE. (1999). Wind tunnel studies of buildings and structures. American Society of Civil Engineers, Reston, VA, U.S.A.

ASCE/SEI. (2013). Minimum Design Loads for Buildings and Other Structures. ASCE standard, American Society of Civil Engineers, Reston, VA, 608.

Bashor, R., and Kareem, A. (2009). "Load factors for dynamically sensitive structures." Proceedings of the11th Americas Conference on Wind Engineering, San Juan, Puerto Rico, June 22-26, CD-ROM.

Beirlant, J., Goegebeur, Y., Teugels, J., and Segers, J. (2004). Statistics of Extremes. Wiley Series in Probability and Statistics, John Wiley \& Sons, Ltd, Chichester, UK.

Castillo, E., Hadi, A. S., Balakrishnan, N., and Sarabia, J. M. (2005). Extreme value and related models with applications in engineering and science. Wiley, New York, NY.

Chen, X., and Huang, G. (2010). "Estimation of probabilistic extreme wind load effects: combination of aerodynamic and wind climate data." Journal of Engineering Mechanics, 136(6), 747-760.

Cherubini, U., Luciano, E., and Vecchiato, W. (2004). Copula Methods in Finance. John Wiley \& Sons Ltd, Oxford, UK.

Coles, S. G., and Tawn, J. a. (1991). "Modelling extreme multivariate events." Journal of the Royal Statistical Society. Series B (Methodological), 53(2), 377-392.

Coles, S. G., and Walshaw, D. (1994). "Directional Modelling of Extreme Wind Speeds." Applied Statistics, 43(1), 139-157. 
Cook, N. J. (1982). “Towards better estimation of extreme winds." Journal of Wind Engineering and Industrial Aerodynamics, 9(3), 295-323.

Cook, N. J., and Mayne, J. R. (1979). "A novel working approach to the assessment of wind loads for equivalent static design." Journal of Wind Engineering and Industrial Aerodynamics, 4(2), 149-164.

Cook, N. J., and Mayne, J. R. (1980). "A refined working approach to the assessment of wind loads for equivalent static design.” Journal of Wind Engineering and Industrial Aerodynamics, 6, 125-137.

Davenport, A. G. (1977). "The prediction of risk under wind loading." Proceedings of the 2nd International Conference on Structural Safety and Reliability, Munich, Germany, September, 1977, $511-538$.

Diniz, S. M. C., Sadek, F., and Simiu, E. (2004). "Wind speed estimation uncertainties: effects of climatological and micrometeorological parameters." Probabilistic Engineering Mechanics, 19(4), $361-371$

Diniz, S. M. C., and Simiu, E. (2005). "Probabilistic descriptions of wind effects and wind-Load factors for database-assisted design.” Journal of Structural Engineering, 131(3), 507-516.

Grigoriu, M. (2007). "Multivariate distributions with specified marginals: applications to wind engineering." Journal of Engineering Mechanics, 133(2), 174-184.

Grigoriu, M. (2009). "Algorithms for generating large sets of synthetic directional wind speed data for hurricane, thunderstorm, and synoptic winds." NIST Technical Note, 1626.

Gumbel, E. J. (1958). Statistics of extremes. Columbia Univ. Press, New York, NY.

Gumley, S. J., and Wood, C. J. (1982). “A discussion of extreme wind-loading probabilities.” Journal of Wind Engineering and Industrial Aerodynamics, 10(1), 31-45.

Haan, L. De, and Ferreira, A. (2007). Extreme value theory: an introduction. Springer, New York, NY.

Hanzlik, P., Diniz, S., Grazini, A., Grigoriu, M., and Simiu, E. (2005). "Building Orientation and Wind Effects Estimation.” Journal of Engineering Mechanics, 131(3), 254-258.

Harris, R. I. (1982). "An improved method for the prediction of extreme values of wind effects on simple buildings and structures." Journal of Wind Engineering and Industrial Aerodynamics, 9(3), 343-379. 
Harris, R. I. (1999). "Improvements to the 'Method of Independent Storms'." Journal of Wind Engineering and Industrial Aerodynamics, 80(1-2), 1-30.

617 Harris, R. I. (2005). "A new direct version of the Cook-Mayne method for wind pressure probabilities in temperate storms." Journal of Wind Engineering and Industrial Aerodynamics, 93(7), 581-600.

Holmes, J. D., and Moriarty, W. W. (1999). “Application of the generalized Pareto distribution to extreme value analysis in wind engineering." Journal of Wind Engineering and Industrial Aerodynamics, 83(1-3), 1-10.

Irwin, P. A., Garber, J., and Ho, E. (2005). "Integration of wind tunnel data with full scale wind climate." Proceedings of the10th Americas Conference on Wind Engineering, Baton Rouge, Louisiana, U.S.A, May 31 - June 4, 132-135.

Isyumov, N., Ho, E., and Case, P. (2014). "Influence of wind directionality on wind loads and responses." Journal of Wind Engineering and Industrial Aerodynamics, Elsevier, 133, 169-180.

Isyumov, N., Mikitiuk, M., Case, P., Lythe, G., and Welburn., A. (2003). "Predictions of wind loads and responses from simulated tropical storm passages." Proceedings of the 11th International Conference on Wind Engineering, Lubbock, Texas, Jun, 2003, CD-ROM.

Itoi, T., and Kanda, J. (2002). "Comparison of correlated Gumbel probability models for directional maximum wind speeds." Journal of Wind Engineering and Industrial Aerodynamics, 90(12-15), $1631-1644$.

Kareem, A. (1987). "Wind effects on structures: a probabilistic viewpoint." Probabilistic Engineering Mechanics, 2(4), 166-200.

Kareem, A. (1988). "Aerodynamic response of structures with parametric uncertainties." Structural Safety, 5(3), 205-225.

Laboy-Rodríguez, S. T., Gurley, K. R., and Masters, F. J. (2014). "Revisiting the directionality factor in ASCE 7." Journal of Wind Engineering and Industrial Aerodynamics, Elsevier, 133, 225-233. 
Lepage, M. F., and Irwin, P. A. (1985). “A technique for combining historical wind data with wind tunnel tests to predict extreme wind loads." Proceedings of the 5th U.S. National Conference on Wind Engineering, Lubbock, Texas, November, 1985, 6-8.

Matsui, M., and Tamura, Y. (2005). "Directional wind speed evaluation in mixed wind climates using modified typhoon simulation method.” Proceedings of International Conference on Structural Safety and Reliability (ICOSSAR 2005), Roma, Italy, 1187-1

Mikosch, T. (2006). “Copulas: Tales and facts.” Extremes, 9, 3-20.192.

Naess, a., and Gaidai, O. (2009). "Estimation of extreme values from sampled time series." Structural Safety, Elsevier Ltd, 31(4), 325-334.

Nelsen, R. B. (2006). An introduction to copulas. Springer, New York, NY.

Renard, B., and Lang, M. (2007). "Use of a Gaussian copula for multivariate extreme value analysis: Some case studies in hydrology." Advances in Water Resources, 30(4), 897-912.

Rigato, A., Chang, P., and Simiu, E. (2001). "Database-assisted design, standardization, and wind direction effects.” Journal of Structural Engineering, 127(8), 855-860.

Sadek, F. (2005). Federal Building and Fire Safety Investigation of the World Trade Center Disaster: Baseline Structural Performance and Aircraft Impact Damage Analysis of the World Trade Center Towers. NIST, NCSTAR 1-2.

Schoelzel, C., and Friederichs, P. (2008). "Multivariate non-normally distributed random variables in climate research - introduction to the copula approach.” Nonlinear Processes Geophysics, 15, 761772.

Simiu, E., and Filliben, J. J. (1981). "Wind direction effects on cladding and structural loads." Engineering Structures, 3, 181-186.

Simiu, E., and Filliben, J. J. (2005). "Wind Tunnel Testing and the Sector-by-Sector Approach to Wind Directionality Effects.” Journal of Structural Engineering, 131(7), 1143-1145.

Simiu, E., and Heckert, N. A. (1996). "Extreme Wind Distribution Tails: A 'Peaks over Threshold' Approach.” Journal of Structural Engineering, 122(5), 539-547. 
Simiu, E., and Heckert, N. A. (1998). "Ultimate wind loads and direction effects in non-hurricane and hurricane-prone regions." Environmetrics, 9(4), 433-444.

Simiu, E., Heckert, N. A., Filliben, J. J., and Johnson, S. K. (2001). "Extreme wind load estimates based on the Gumbel distribution of dynamic pressures: an assessment.” Structural Safety, 23(3), 221-229.

Simiu, E., Letchford, C., Isyumov, N., Chowdhury, A. G., Yeo, D. (2013). “Assessment of ASCE 7-10 standard methods for determining wind loads." Journal of Structural Engineering, 139(11), 20442047.

Simiu, E., and Scanlan, R. H. (1996). Wind Effects on Structures: Fundamentals and Application to Design. John Wiley \& Sons, New York, NY.

Wen, Y. (1983). "Wind Direction and Structural Reliability." Journal of Structural Engineering, 109(4), $1028-1041$.

Wen, Y. (1984). "Wind Direction and Structural Reliability: II.” Journal of Structural Engineering, 110(6), 1253-1264.

Xu, Z., Bekele, S., Mikitiuk, M., Isyumov, N., and Ho, E. (2008). "Bi-modal Weibull distribution fit for climatic wind speed histogram." Proceedings of the13th Australasian Wind Engineering Society Workshop, Hobart, Australia, December 4-5, 32-35.

681 Zhang, X. (2015). Estimation of probabilistic extreme wind load effect with consideration of directionality and uncertainty. Submitted to graduate faculty of Texas Tech University in partial fulfillment of the requirements for the Degree of Philosophy. 
1

2

3

4685

5

7

8

9

10

11

12

13

14

15

16

17

18686

19687

20688

23

24

25

26

27

28

29

30

31

32

33

34

35

36

37

38

39

40

41

42

43

44

45

46

47

48

49

50

51

52

53

54

55

56

57

58

59

60

61

62

63

64

65

Table 1. Estimated wind speeds for each direction and regardless of direction

\begin{tabular}{|c|c|c|c|c|c|c|c|c|c|c|c|c|}
\hline \multirow[b]{2}{*}{ Model } & \multirow[b]{2}{*}{ Margin } & \multirow{2}{*}{$\begin{array}{c}\text { MRI } \\
\text { (years) }\end{array}$} & \multicolumn{8}{|c|}{$v_{i, R}(\mathrm{mph})$} & \multicolumn{2}{|c|}{$v_{R}(\mathrm{mph})$} \\
\hline & & & $\mathrm{E}$ & $\mathrm{NE}$ & $\mathrm{N}$ & NW & W & SW & $S$ & $\mathrm{SE}$ & $\begin{array}{l}v_{R, \text { non }} \\
\text { data fit }\end{array}$ & $\begin{array}{c}\text { Multivariate } \\
\text { model }\end{array}$ \\
\hline \multirow{4}{*}{$U$-model } & \multirow{2}{*}{ Type I } & 50 & 47.8 & 48.8 & 40.9 & 58.9 & 67.3 & 47.0 & 34.6 & 40.2 & 65.5 & 67.9 \\
\hline & & 500 & 57.9 & 59.2 & 48.4 & 70.9 & 81.5 & 56.0 & 41.2 & 48.5 & 78.1 & 81.9 \\
\hline & \multirow{2}{*}{ GEV } & 50 & 46.0 & 45.7 & 33.1 & 45.5 & 56.5 & 39.9 & 31.2 & 42.7 & 55.5 & 56.8 \\
\hline & & 500 & 54.2 & 52.8 & 34.3 & 47.1 & 60.9 & 42.6 & 34.6 & 54.4 & 59.3 & 61.7 \\
\hline \multirow{4}{*}{$Q$-model } & \multirow{2}{*}{ Type I } & 50 & 42.2 & 43.2 & 37.0 & 51.6 & 58.8 & 42.1 & 31.2 & 36.0 & 58.6 & 58.9 \\
\hline & & 500 & 47.7 & 48.9 & 41.3 & 58.0 & 66.4 & 47.3 & 35.0 & 40.7 & 65.8 & 66.5 \\
\hline & \multirow{2}{*}{ GEV } & 50 & 51.0 & 47.8 & 34.5 & 47.9 & 60.9 & 41.9 & 32.9 & 45.1 & 57.9 & 62.3 \\
\hline & & 500 & 66.0 & 59.0 & 36.9 & 51.5 & 70.2 & 46.9 & 38.5 & 60.8 & 64.4 & 73.8 \\
\hline
\end{tabular}


Table 2. Covariance Matrices of directional wind speeds and Gaussian variables 
Table 3. Normalized wind load effect $x_{i, R} / x_{w, 500}$ in each directions, Type I wind speed margins

\begin{tabular}{|c|c|c|c|c|c|c|c|c|c|c|c|}
\hline \multicolumn{4}{|c|}{ Directions } & $\mathrm{E}$ & $\mathrm{NE}$ & $\mathrm{N}$ & NW & $\mathrm{W}$ & SW & $S$ & SE \\
\hline \multirow{10}{*}{ Example 1} & \multirow{2}{*}{ Model } & \multirow{2}{*}{ b } & \multirow{2}{*}{$\begin{array}{c}\text { MRI } \\
\text { (years) }\end{array}$} & \multicolumn{8}{|c|}{$C_{\alpha i}$} \\
\hline & & & & 1.00 & 0.32 & 0.02 & 0.07 & 0.34 & 0.20 & 0.20 & 0.15 \\
\hline & \multirow{4}{*}{$U$-model } & \multirow{2}{*}{2} & 50 & 0.341 & 0.113 & 0.005 & 0.037 & 0.227 & 0.065 & 0.036 & 0.037 \\
\hline & & & 500 & 0.500 & 0.166 & 0.007 & 0.054 & 0.333 & 0.093 & 0.051 & 0.054 \\
\hline & & \multirow{2}{*}{3} & 50 & 0.199 & 0.067 & 0.003 & 0.027 & 0.186 & 0.037 & 0.015 & 0.018 \\
\hline & & & 500 & 0.354 & 0.120 & 0.004 & 0.047 & 0.331 & 0.063 & 0.026 & 0.032 \\
\hline & \multirow{4}{*}{$Q$-model } & \multirow{2}{*}{2} & 50 & 0.404 & 0.134 & 0.007 & 0.043 & 0.263 & 0.080 & 0.044 & 0.045 \\
\hline & & & 500 & 0.516 & 0.172 & 0.008 & 0.055 & 0.336 & 0.100 & 0.056 & 0.058 \\
\hline & & \multirow{2}{*}{3} & 50 & 0.256 & 0.087 & 0.004 & 0.034 & 0.233 & 0.050 & 0.021 & 0.024 \\
\hline & & & 500 & 0.371 & 0.127 & 0.005 & 0.048 & 0.336 & 0.071 & 0.029 & 0.035 \\
\hline \multirow{10}{*}{ Example 2} & \multirow{2}{*}{ Model } & \multirow{2}{*}{ b } & \multirow{2}{*}{$\begin{array}{c}\text { MRI } \\
\text { (years) }\end{array}$} & \multicolumn{8}{|c|}{$C_{\alpha i}$} \\
\hline & & & & 0.80 & 1.00 & 0.80 & 0.60 & 0.80 & 1.00 & 0.80 & 0.60 \\
\hline & \multirow{4}{*}{$U$-model } & \multirow{2}{*}{2} & 50 & 0.273 & 0.354 & 0.200 & 0.310 & 0.540 & 0.329 & 0.142 & 0.144 \\
\hline & & & 500 & 0.400 & 0.523 & 0.279 & 0.449 & 0.791 & 0.468 & 0.202 & 0.210 \\
\hline & & \multirow{2}{*}{3} & 50 & 0.159 & 0.211 & 0.100 & 0.223 & 0.443 & 0.189 & 0.060 & 0.071 \\
\hline & & & 500 & 0.283 & 0.378 & 0.165 & 0.389 & 0.787 & 0.320 & 0.102 & 0.124 \\
\hline & \multirow{4}{*}{$Q$-model } & \multirow{2}{*}{2} & 50 & 0.324 & 0.425 & 0.243 & 0.359 & 0.628 & 0.397 & 0.174 & 0.177 \\
\hline & & & 500 & 0.413 & 0.541 & 0.309 & 0.458 & 0.799 & 0.506 & 0.222 & 0.225 \\
\hline & & \multirow{2}{*}{3} & 50 & 0.205 & 0.274 & 0.138 & 0.280 & 0.555 & 0.254 & 0.082 & 0.096 \\
\hline & & & 500 & 0.297 & 0.398 & 0.192 & 0.400 & 0.799 & 0.360 & 0.117 & 0.138 \\
\hline
\end{tabular}


50

51

52

53

54

55

56

57

58

59

60

61

62

Table 4. Normalized wind load effects $x_{R} / x_{w, 500}$ regardless of direction, example 1 Type I wind speed margins.

\begin{tabular}{c|c|cc|cc}
\hline \hline \multicolumn{2}{c|}{ Structure parameter } & \multicolumn{2}{c|}{$\mathrm{b}=2$} & \multicolumn{2}{c}{$\mathrm{b}=3$} \\
\hline \multicolumn{2}{c}{ MRI (year) } & 50 & 500 & 50 & 500 \\
\hline \multirow{2}{*}{ Existing } & $U$-model & 0.324 & 0.464 & 0.207 & 0.348 \\
& $Q$-model & 0.262 & 0.331 & 0.150 & 0.212 \\
\hline \multirow{2}{*}{ Proposed } & $U$-model & 0.346 & 0.504 & 0.232 & 0.399 \\
& $Q$-model & 0.266 & 0.340 & 0.148 & 0.209 \\
\hline \hline
\end{tabular}


Table 5. Normalized wind load effects $x_{R} / x_{w, 500}$ regardless of direction with consideration of uncertainty

\begin{tabular}{|c|c|c|c|c|c|c|c|c|c|c|}
\hline \multirow{2}{*}{$C_{01}(\alpha)$} & \multirow{2}{*}{ b } & \multirow{2}{*}{ Directionality } & \multirow{2}{*}{ MRI } & \multicolumn{5}{|c|}{$V_{c i}=V_{c}$} & \multirow{2}{*}{$V_{c 1}(\alpha)$} & \multirow{2}{*}{$V_{c 2}(\alpha)$} \\
\hline & & & & 0 & 0.1 & 0.2 & 0.3 & 0.4 & & \\
\hline \multirow{8}{*}{ Example 1} & \multirow{4}{*}{2} & \multirow{2}{*}{ Worst case } & 50 & 0.686 & 0.697 & 0.737 & 0.793 & 0.878 & 0.886 & 0.689 \\
\hline & & & 500 & 1.000 & 1.022 & 1.103 & 1.207 & 1.401 & 1.437 & 1.005 \\
\hline & & \multirow{2}{*}{ Directional } & 50 & 0.346 & 0.352 & 0.372 & 0.403 & 0.451 & 0.440 & 0.367 \\
\hline & & & 500 & 0.504 & 0.515 & 0.556 & 0.611 & 0.712 & 0.713 & 0.543 \\
\hline & \multirow{4}{*}{3} & Worct sose & 50 & 0.569 & 0.573 & 0.591 & 0.621 & 0.665 & 0.669 & 0.570 \\
\hline & & Worst case & 500 & 1.000 & 1.012 & 1.058 & 1.127 & 1.242 & 1.258 & 1.003 \\
\hline & & Diroctionel & 50 & 0.232 & 0.234 & 0.244 & 0.258 & 0.280 & 0.259 & 0.251 \\
\hline & & Directional & 500 & 0.397 & 0.404 & 0.425 & 0.457 & 0.510 & 0.468 & 0.450 \\
\hline \multirow{8}{*}{ Example 2} & \multirow{4}{*}{2} & \multirow{2}{*}{ Worst case } & 50 & 0.690 & 0.736 & 0.821 & 0.947 & 1.093 & 0.880 & 0.891 \\
\hline & & & 500 & 1.000 & 1.077 & 1.212 & 1.420 & 1.674 & 1.332 & 1.348 \\
\hline & & \multirow{2}{*}{ Directional } & 50 & 0.549 & 0.563 & 0.598 & 0.666 & 0.723 & 0.575 & 0.693 \\
\hline & & & 500 & 0.796 & 0.823 & 0.887 & 1.009 & 1.133 & 0.837 & 1.088 \\
\hline & \multirow{4}{*}{3} & \multirow{2}{*}{ Worst case } & 50 & 0.569 & 0.606 & 0.668 & 0.759 & 0.858 & 0.704 & 0.711 \\
\hline & & & 500 & 1.000 & 1.069 & 1.186 & 1.359 & 1.588 & 1.263 & 1.277 \\
\hline & & \multirow{2}{*}{ Directional } & 50 & 0.453 & 0.460 & 0.478 & 0.513 & 0.533 & 0.461 & 0.525 \\
\hline & & & 500 & 0.790 & 0.809 & 0.848 & 0.928 & 0.986 & 0.804 & 0.970 \\
\hline
\end{tabular}




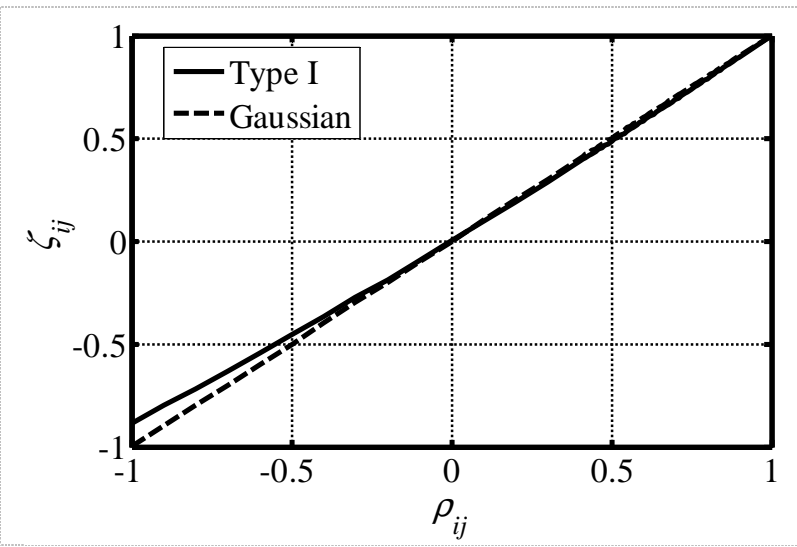

Fig. 1. Relationship between correlation coefficients of Type I and its underlying Gaussian variables 


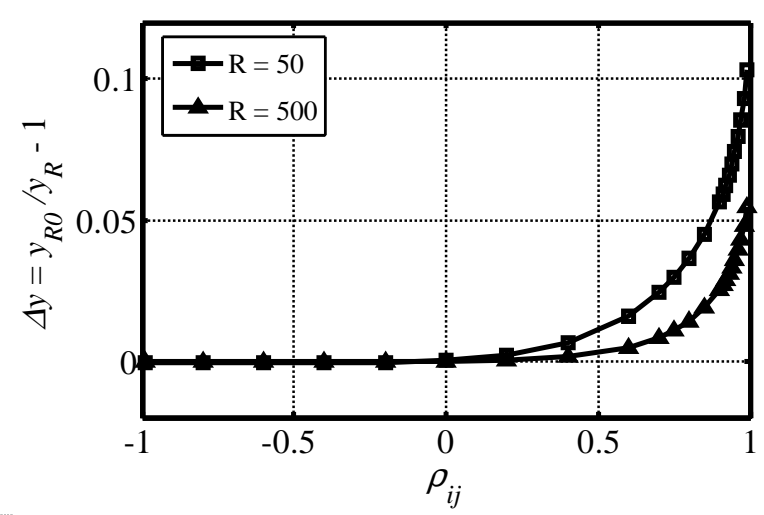

Fig. 2. Difference of bivariate Gaussian values between $\Sigma_{i j}=\rho_{i j}$ and $\Sigma_{i j}=0$ 


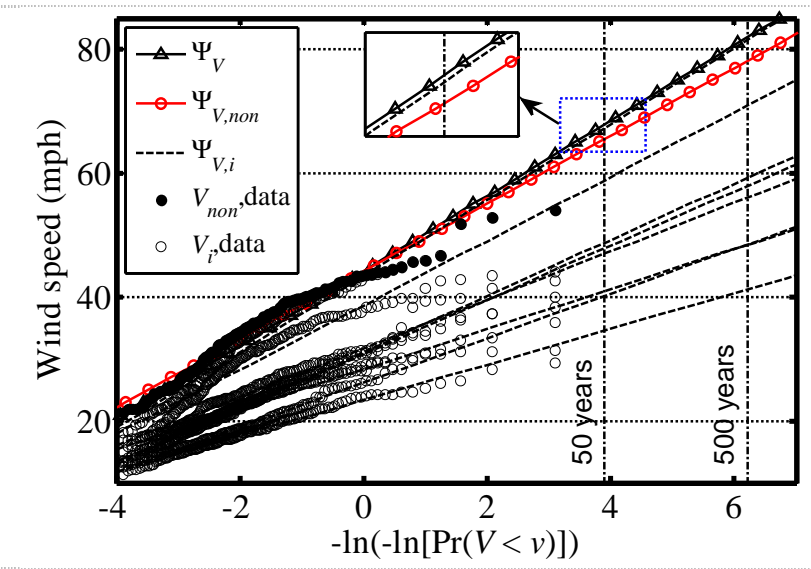

(a) Type I margins

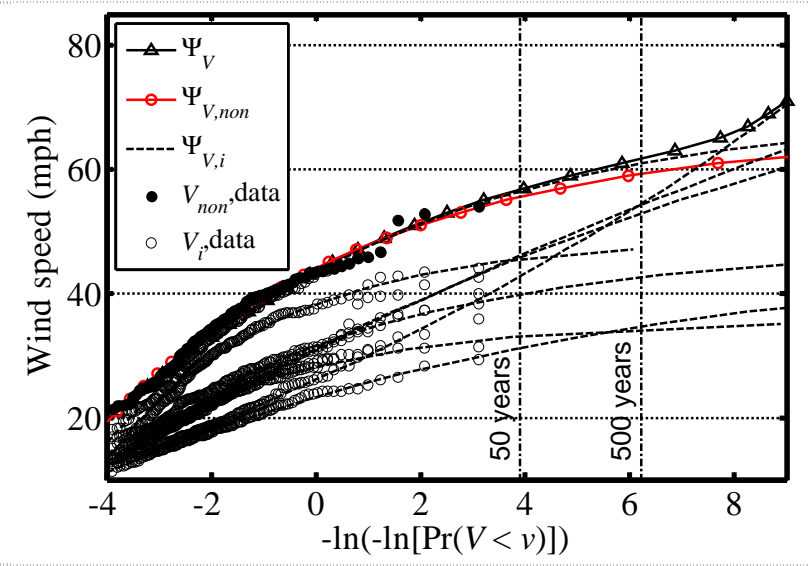

(b) GEV margins 


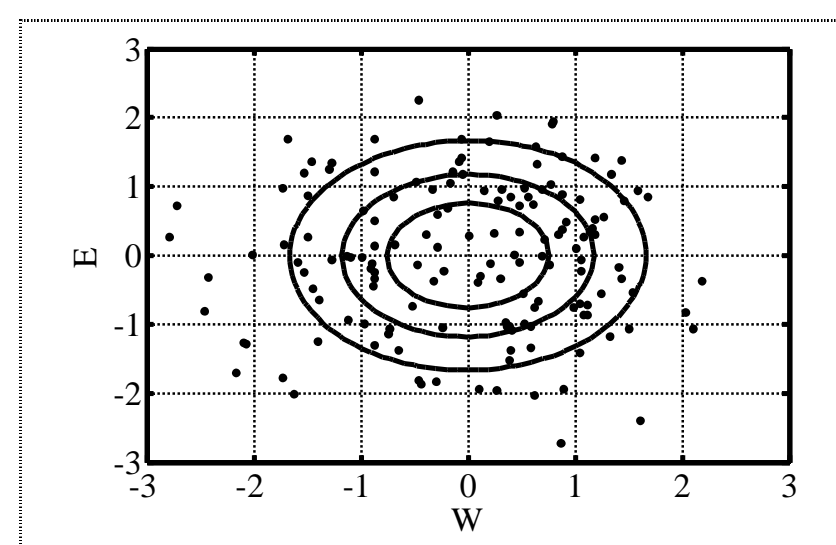

(a) Gaussian variables in $\mathrm{E}$ and $\mathrm{W}$ directions

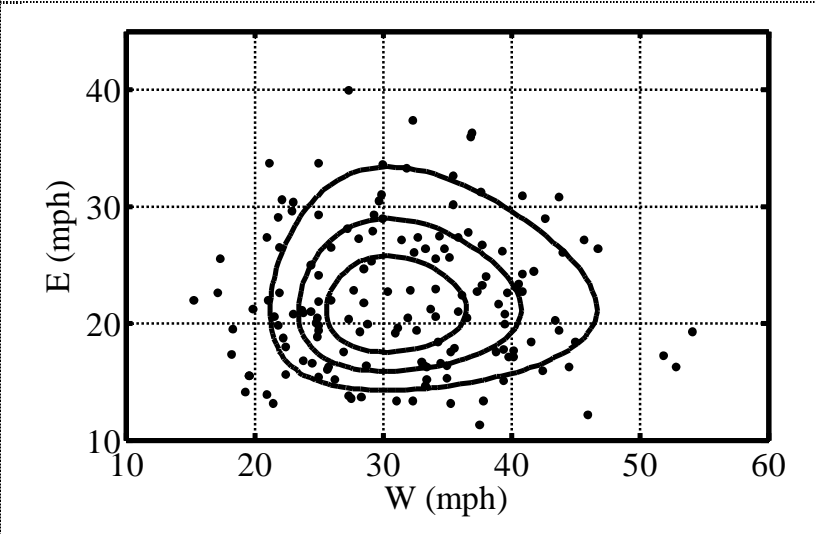

(c) Wind speeds in $\mathrm{E}$ and $\mathrm{W}$ directions

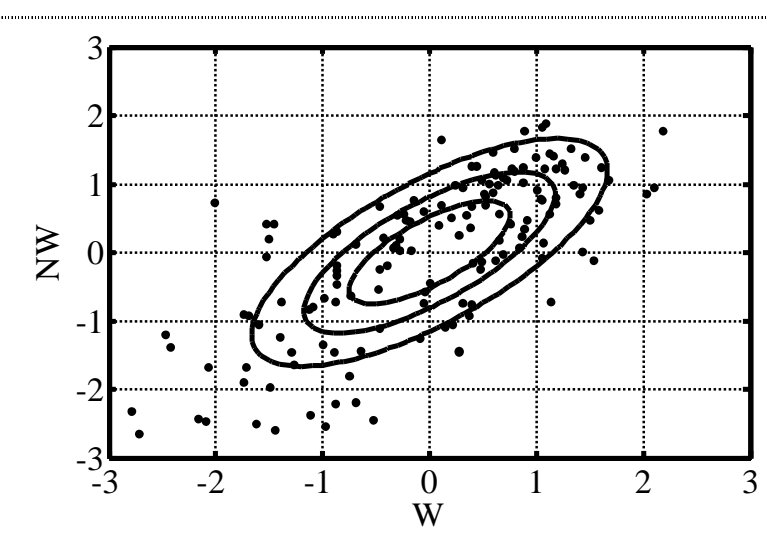

(b) Gaussian variables in NW and $\mathrm{W}$ directions

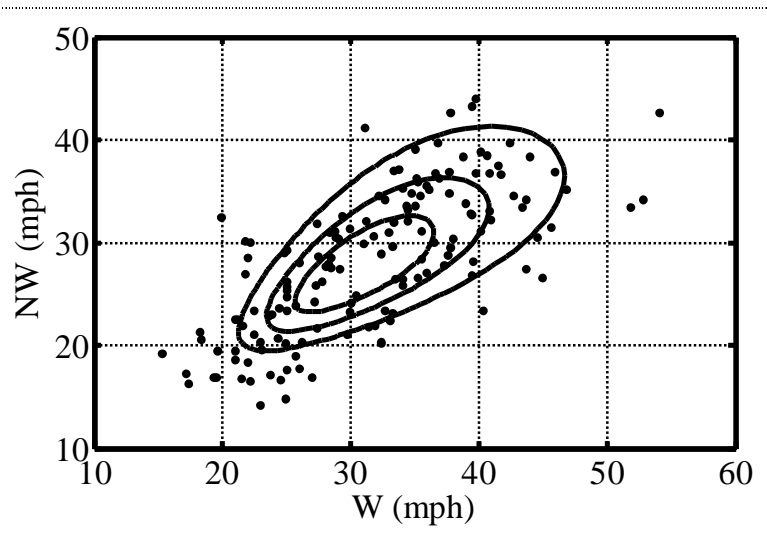

(d) Wind speeds in NW and W directions 


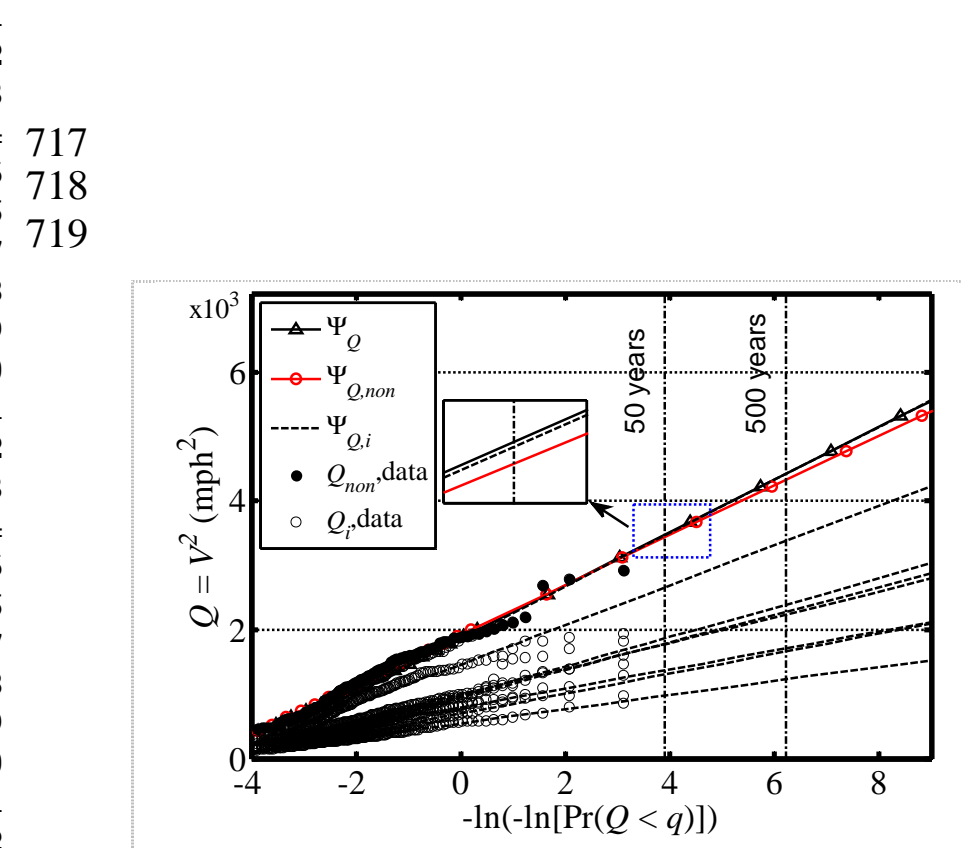

(a) Type I margins

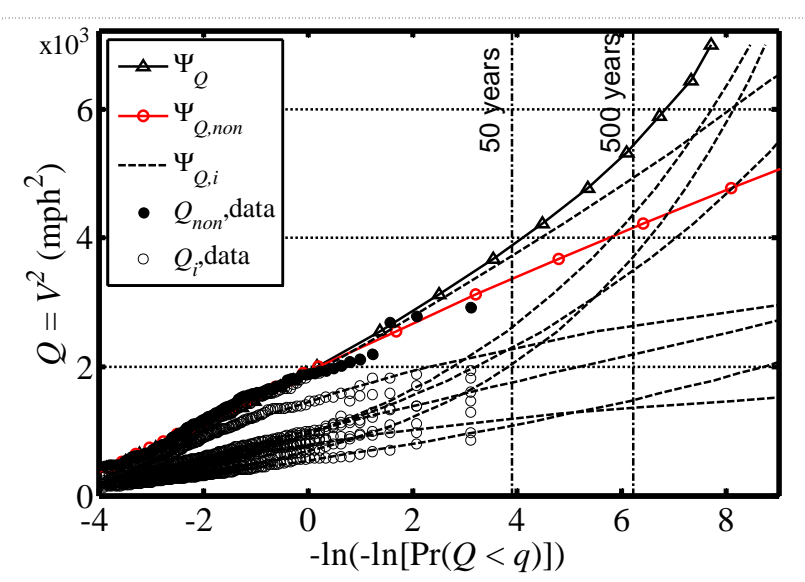

(b) GEV margins

Fig. 5. Multivariate extreme wind climate model with different margin types ( $Q$-model) 


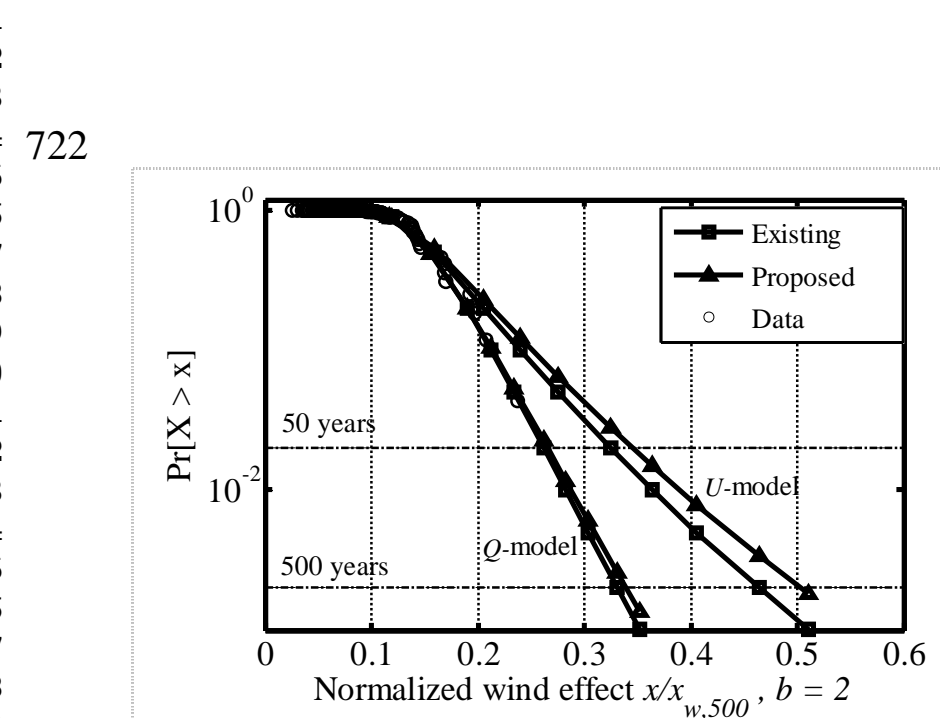

(a) $b=2$

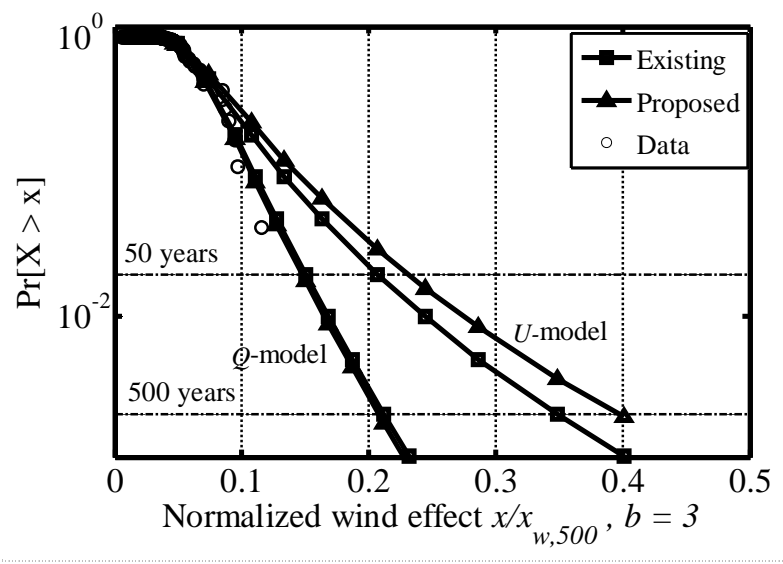

(b) $b=3$

723 Fig. 6 Distribution of directionless wind load effect predicted from $U$ - and $Q$-model for both rigid and 22724 23725 


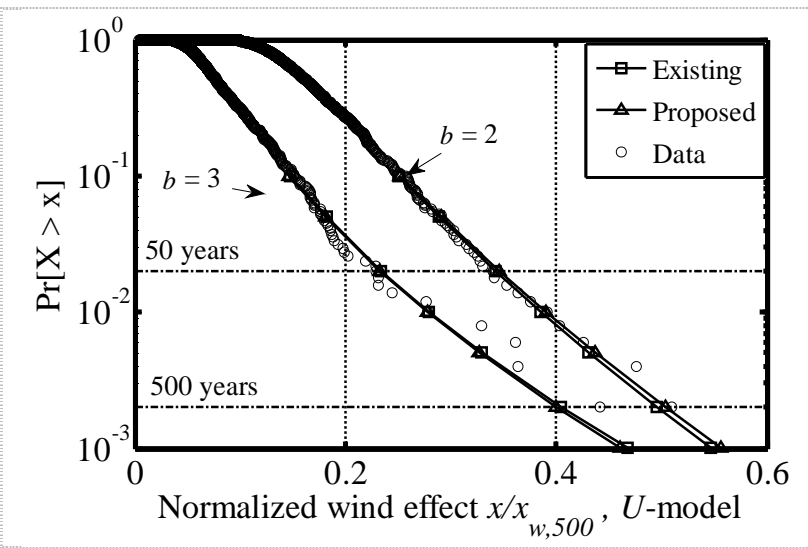

Fig. 7. Comparison of wind load effect distributions 


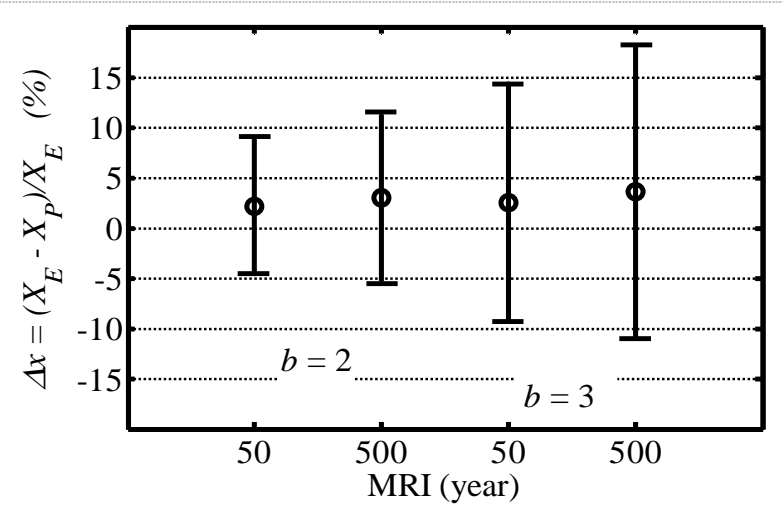

Fig. 8. Differences of predicted 50- and 500-year wind load effects with sample size 150 


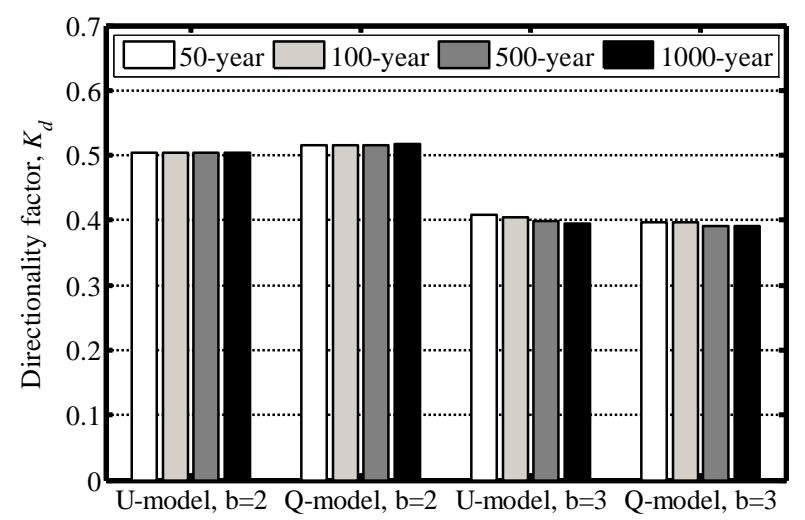

(a) Example 1

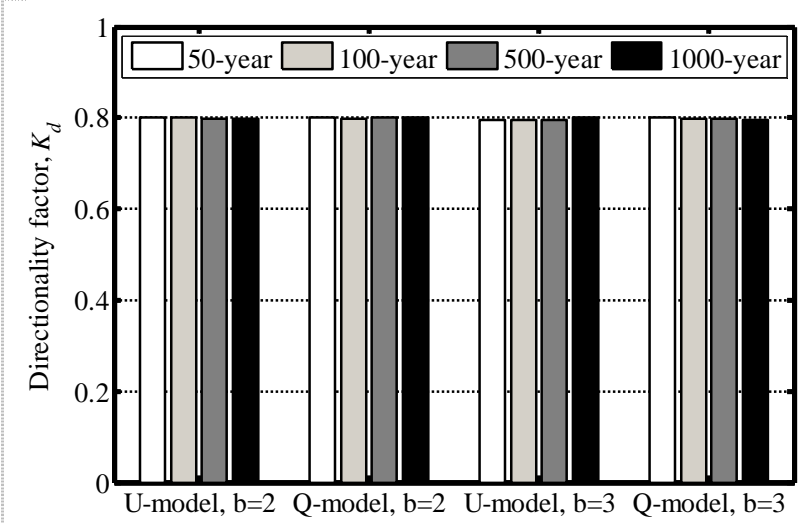

(b) Example 2

Fig. 9. $K_{d}$ as a function of MRI for Q- and U- models and for rigid and flexible structures 


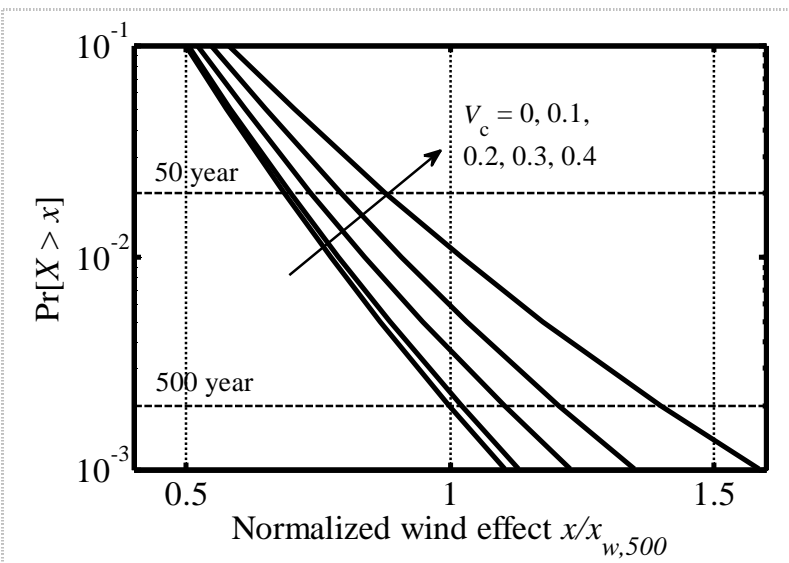

(a) Worst case

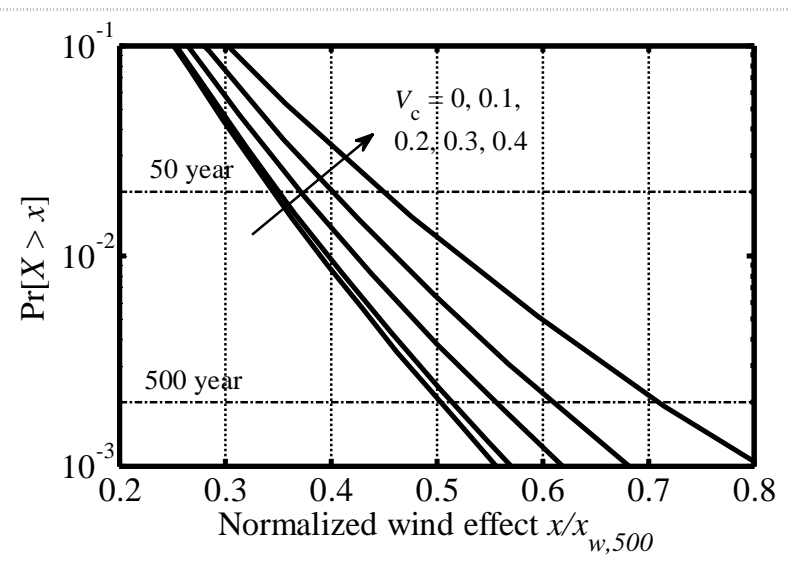

(b) Directional

Fig. 10. Distribution of wind load effect with $V_{c}=0,0.1,0.2,0.3$ and 0.4 (Example 1, $b=2$ ) 
1

2

3

4739

6740

8

9

10

11

12

13

14

15

16

17

18

19

20

21

22

23

24741

25

26742

27

28

29

30

31

32

33

34

35

36

37

38

39

40

41

42

43

44

45

46

47

48

49

50

51

52

53

54

55

56

57

58

59

60

61

62

63

64

65

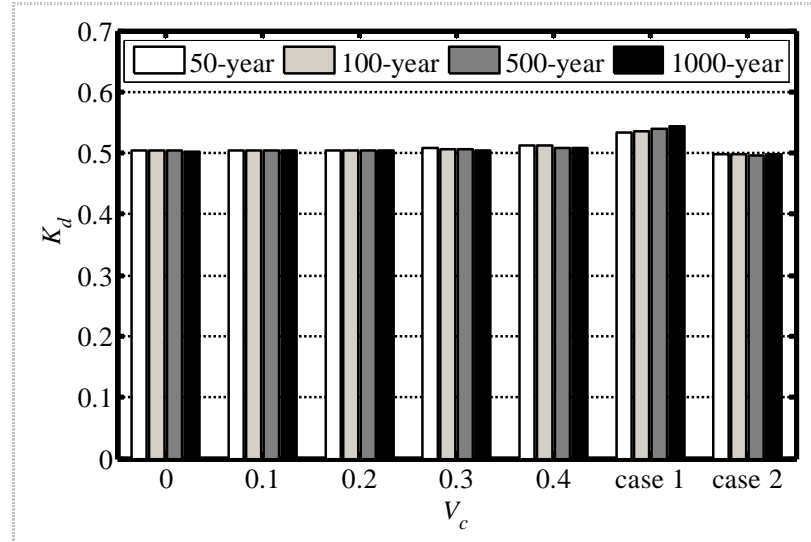

(a) Example 1

Fig. 11 Influence of uncertainty of wind load coefficient on directionality factor $K_{d}(b=2)$ 


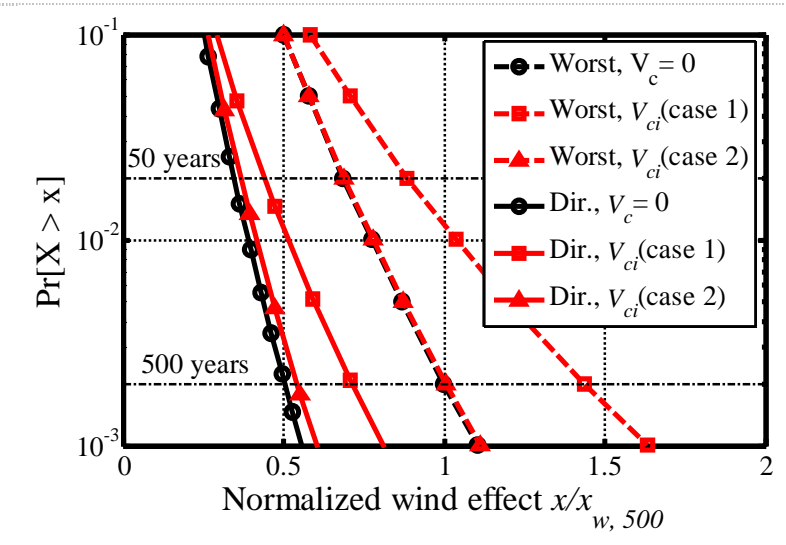

(a) Example 1

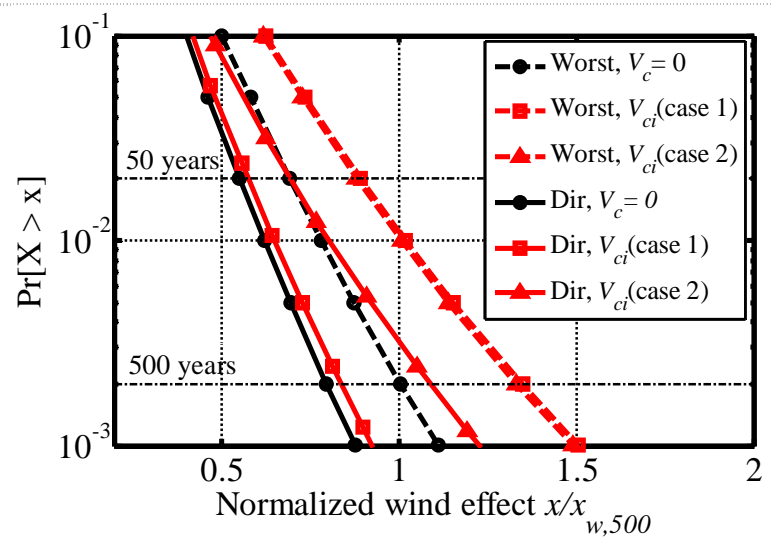

(b) Example 2

Fig. 12. Distribution of directional wind load effect with $V_{c}$ as a function of wind direction $(\mathrm{b}=2)$ 